\title{
Externally Pressurized Thrust Spherical Bearing Performance Under Variable Lubricant Viscosity (Un-recessed Clearance Type)
}

\author{
Ahmad Waguih Yacout Elescandarany \\ Mechanical Department, Faculty of Engineering, Alexandria University, Alexandria, Egypt \\ Email address: \\ awaguih@yahoo.com

\section{To cite this article:} \\ Ahmad Waguih Yacout Elescandarany. Externally Pressurized Thrust Spherical Bearing Performance under Variable Lubricant Viscosity \\ (Un-recessed Clearance Type). International Journal of Mechanical Engineering and Applications. Vol. 8, No. 2, 2020, pp. 45-64. \\ doi: 10.11648/j.ijmea.20200802.11
}

Received: March 19, 2020; Accepted: April 7, 2020; Published: April 28, 2020

\begin{abstract}
In a previous study handling this subject, a series of six papers has been published covering the fitted type (equal radii of this sort of bearings) with its configurations in details. The present study handles the same subject investigating the clearance type of this sort of bearings starting with the un-recessed one. The clearance type of bearings, where the sphere radius is smaller than that of the seat, provides plenty of complications and great divergence in its behavior compared with the fitted one. The study investigates theoretically the bearing behavior under the lubricant viscosity variation in the presence of the centripetal inertia due to the shaft rotation and the surface roughness. Solutions have been derived for the un-recessed clearance type of bearings with hemispherical and partial hemispherical seats in addition to its two special cases using capillary tube and orifice restrictors. Unlike other studies, using the conventional integration (i.e., without using the Sommerfeld substitution), only one equation with one form for the pressure gradient is derived to cover the positive and negative eccentricity ratios. Expressions for the pressure distribution, temperature distribution in turn the temperature rise, load carrying capacity; volume flow rate, frictional torque, friction factor, power losses and stiffness factor are obtained. The study shows the combined effects of the viscosity variation, the centripetal inertia and the surface roughness on the bearing performance. The optimum design (with constant viscosity) based on the minimum power losses, minimum flow rate and the optimal restrictor dimensions, in a previous study is checked where it is found out that designing this type of bearings on such basis only is not sufficient in despite of the bearing consistency. A natural dynamic phenomenon ignored in the previous study is revealed and briefly touched.
\end{abstract}

Keywords: Externally Pressurized Bearings, Spherical Bearings, Surface Roughness, Hydrostatic Bearing's Design, Effect of Rotational Inertia, Bearing Temperature Rise

\section{Introduction}

Because of their fabrication simplicity, potential characteristics of low running friction, high load carrying capacity, high stiffness, small viscous dissipation, self aligning property and ability to accept thrust and radial loads, the externally pressurized spherical bearings are preferably used in heavy duty and high speed devices as machine tools, gyroscope gimbals, radar tracking units, craft engines, dynamometers and telescopes. Where the manufacture processes in forming and finishing stages consider all bearing surfaces rough in engineering practice, the recent researches studied the effect of the surface roughness on the bearing behavior.

Yacout [1-4] studied the combined effects of the surface roughness and the centripetal inertia on the hydrostatic thrust spherical bearing performance (fitted and clearance) where it is concluded that the central pressure ratio should be $(2 / 3)$ showing the bearing behavior in details and offering an example of a bearing designed on the minimum power losses, minimum flow rate and optimal restrictor dimensions. The effect of the viscosity variability on fitted type bearing behavior in the presence of the surface roughness and the centripetal inertia has been studied showing the temperature distribution and calculating the temperature rise through introducing the energy equation. The aforementioned 
example of the designed bearing was checked showing that the temperature rise is less than 14 degrees centigrade in addition to the bearing consistency which proved the reliability of the design technique [2-3]. The clearance type (un-recessed) bearing behavior under the surface roughness and the centripetal inertia has been studied where a single equation with two forms for the pressure is derived to cover the negative and positive eccentricity ratios, in turn a single equation with two forms for the load [4].

Woonsil Lee et al [5] offered an actual fruitful industrial case for a high bearing temperature troubleshooting of centrifugal heat-pump compressor where the bearing variables were studied practically (online) finding out considerable differences between the actual operating data and those submitted by the designer.

Yongping SUN, Minghui HAO, Baoyu SONG [6] numerically studied the load capacity and temperature rise of herringbone groove hydrodynamic lubricating bearing showing the effects of the rotational speed and the eccentricity ratio on the load and the temperature rise.

Srinivasan [7, 8] studied, the annular recessed bearing considering the inertia and the viscosity variation concluding that the results show that oil cavity pressure is almost invariant by increasing the workbench rotating velocity while the oil cavity temperature is increasing gradually by increasing the workbench rotating velocity. Along with the work table rotational speed increasing, the turbulent flow in the oil cavities is more and more obvious. The environmental temperature around a power transmission system is equally important because it establishes the stabilized base temperature for the system.

Shigang Wang et al [9] studied the temperature effect on the hydrostatic bearing performance in both cases of sufficient and deficient fluid film using the "Fluent 6.5 Software" to simulate the temperature field. According to the obtained results, it is concluded that the oil will affect the temperature distribution and the temperature rise.

Xibing Li et al [10] studied heavy/large hydrostatic thrust bearing concluding that, during working, the viscosity changes as the temperature increases hence the bearing performance will be affected; therefore control thermal deformation of hydrostatic bearing and high efficiency cooling is the prime problem to solve, in turn an optimal design of the oil chamber to produce the least amount of heat is necessary.

Naduvinamani and Kadadi [11] studied the effect of the viscosity variation on a short journal bearing behavior stating that the effect of the variation in the viscosity leads to decrease the load carrying capacity.

Bouchehit et al [12] studied a foil lubricated bearing and concluded that the temperature has a noticeable effect on the bearing performance.

Yuan Kang et al [13] studied the stiffness of open and closed types of hydrostatic plain bearings with constant compensations to find out the most appreciable technique for designing a stiffened bearing concluding that the static stiffness of open-type plain bearing is function of the recess pressure and the compensations of both constant restrictions of orifice and capillary correspond to low stiffness in the range of recess pressure ratio $<0.4$ or $>0.8$.

G Jaya Chandra et al [14] theoretically studied the effect of the viscosity variation on the squeeze film performance of a short bearing operating with couple stress fluids finding that the load carrying capacity decreases with the decreasing the viscosity variation factor.

Jun Sun [15] studied the effect of surface roughness, viscosity and elastic deformation on lubrication performance of misaligned journal bearings finding that the surface directional parameter has an obvious effect on the lubrication performance of the bearings when the ratio of the minimum film thickness to the surface roughness is small.

Chuan-Chieh et al [16] numerically studied the effects of lubricant viscosity ratio on the hydrostatic thrust bearing performance concluding that excellent bearing characteristics could be obtained from the relationship between effective area, supply pressure, restriction constants of restrictor and bearing pad, and specific value of average viscosity of the lubricant; And when changing viscosity ratio (with fixing the other design parameters) the simulation results depict that the lubricant viscosity during restrictor being greater than that in bearing pad film will make the load capacity decrease, but will improve the film static stiffness.

Due to the rare works that handles such type of bearings in details, theoretically or experimentally, the present research continues the investigation of the hydrostatic thrust spherical bearing in work [4] where the study offers an attempt to find out the bearing thermal behavior under variable viscosity of the lubricant fluid in presence of centripetal inertia and surface roughness. The solutions are presented for the unrecessed clearance type of bearings (different radii) with hemispherical and partial hemispherical seats using capillary tube and orifice restrictors. The flow of the lubricant is steady and the fluid viscosity has been treated as a variable in the flow direction.

\section{Theoretical Analysis}

The modified form of Reynolds equation derived by Yacout [1-4] and the suggested equation for the lubricant viscosity variation applicable to the hydrosphere figure 1 have been adopted with little deviation in this study.

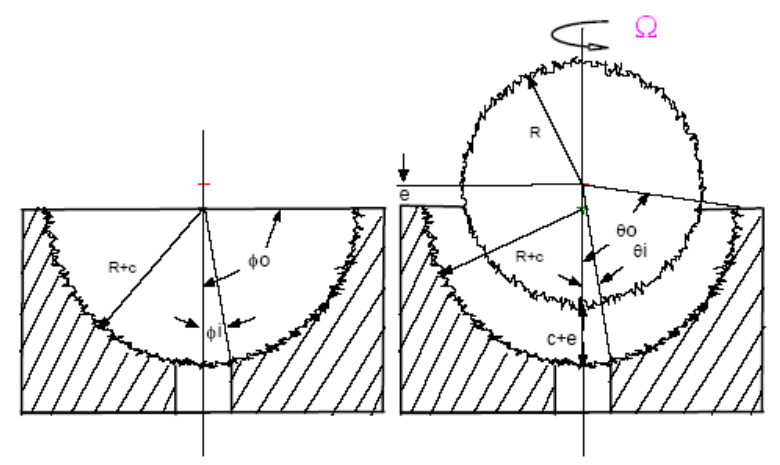

Figure 1. Bearing configuration. 


\section{Theoretical Solution}

\subsection{Pressure Distribution}

\subsubsection{The Case of $( \pm \mathcal{E})$}

From appendix (A1.1):

$$
\begin{aligned}
P & =A\left\{a_{1} \ln (H)+\frac{c_{1}}{2} \ln \left(H-\alpha_{1}\right)^{2}\right. \\
& +\frac{d_{1}}{2} \ln n\left(H-\beta_{1}\right)^{2}+\frac{e_{1}}{2} \ln \left(H^{2}+b^{2}\right\}+\frac{f_{1}}{b} \tan ^{-1}\left(\frac{H}{b}\right) \\
& \left.-\left(\frac{K_{v}}{8 \varepsilon^{6}}\right)\left[a_{1 v} \ln (H)+\frac{e_{1 v}}{2} \ln \left(H^{2}+b^{2}\right)+\frac{f_{1 v}}{b} \tan ^{-1}\left(\frac{H}{b}\right)\right]\right\} \\
& -S \cos (2 \theta)+B
\end{aligned}
$$

\subsubsection{The First Exceptional Case $(\varepsilon=0)$}

From appendix (A1.2):

$$
P=\frac{A}{1+b^{2}}\left[\ln \left(\frac{\sin \theta}{1+\cos \theta}\right)+K_{v} \cos \theta\right]-S \cos (2 \theta)+B
$$

\subsubsection{The Second Exceptional Case $(\varepsilon=1)$}

From appendix (A1.3):

$$
\begin{aligned}
P & =A\left\{\frac{1}{2 b^{2} H}-\left(\frac{1}{4 b^{2}}\right) \ln (H)+\frac{1}{4\left(4+b^{2}\right)} \ln (2-H)\right. \\
& +\frac{1}{2 b^{2}\left(4+b^{2}\right)} \ln \left(H^{2}+b^{2}\right)+\frac{2}{b^{3}\left(4+b^{2}\right)} \tan ^{-1}\left(\frac{H}{b}\right) \\
& \left.+K_{v}\left[a_{v} \ln (H)+\frac{e_{v}}{2} \ln \left(H^{2}+b^{2}\right)\right]\right\}-S \cos (2 \theta)+B
\end{aligned}
$$

\subsubsection{Dynamic Pressure Phenomenon}

From appendix (A1.3):

$$
\begin{array}{ll}
P_{D}=A\left[c_{1} \ln \left(H-\alpha_{1}\right)\right] & \text { for }(+\varepsilon) \\
P_{D}=A\left[d_{1} \ln \left(H-\beta_{1}\right)\right] & \text { for }(-\varepsilon)
\end{array}
$$

\subsection{Load Carrying Capacity}

\subsubsection{The Case of $( \pm \varepsilon)$}

From appendix (A2.1):

$$
\begin{aligned}
W= & \sin ^{2} \theta_{i}+2 A\left[\left(a_{1} w_{1}+c_{1} w_{2}+d_{1} w_{3}+\frac{e_{1}}{2} w_{4}+\frac{f_{1}}{b} w_{5}\right)\right. \\
& \left.-\left(\frac{-K_{v}}{\varepsilon^{2}}\right)\left(a_{1 v} I_{1}+\frac{e_{1 v}}{2} I_{2}\right)\right]_{\theta_{i}}^{\theta_{o}}+\frac{S}{4}\left[\cos ^{2} 2 \theta\right]_{\theta_{i}}^{\theta_{e}}+B\left[\sin ^{2} \theta\right]_{\theta_{i}}^{\theta_{e}}
\end{aligned}
$$

\subsubsection{The First Exceptional Case $(\varepsilon=0)$}

From appendix (A2.2):

$$
\begin{aligned}
& W=\sin ^{2} \theta_{l}+\left(\frac{A}{1+b^{2}}\right)\left[\sin ^{2} \theta \ln \left(\frac{\sin \theta}{1+\cos \theta}\right)+\cos \theta-\frac{1}{2}\right. \\
& \left.-\left(\frac{2}{3}\right) K_{v} \cos ^{3}(2 \theta)\right]_{\theta_{i}}^{\theta_{o}}+\frac{S}{4}[\cos (2 \theta)]_{\theta_{i}}^{\theta_{o}}+B\left[\sin ^{2} \theta\right]_{\theta_{i}}^{\theta_{o}}
\end{aligned}
$$

\subsubsection{The Second Exceptional Case $(\varepsilon=1)$}

From appendix (A2.3):

$$
\begin{aligned}
W= & \sin ^{2} \theta_{i}+2 A\left\{a_{1} w_{1}+a_{2} w_{2}+a_{3} w_{3}+a_{4} w_{4}+a_{5} w_{5}\right. \\
& \left.-K_{v}\left[a_{v} I_{1 v}+\frac{e_{v}}{2} I_{2 v}\right]\right\}_{\theta_{i}}^{\theta_{o}}+\frac{S}{4}[\cos (2 \theta)]_{\theta_{i}}^{\theta_{o}}+B\left[\sin ^{2} \theta\right]_{\theta_{i}}^{\theta_{o}}
\end{aligned}
$$

\subsubsection{Dynamic Load Phenomenon}

From appendix (A2.4):

$$
\begin{array}{ll}
L_{d}=2 A\left(c_{1} w_{2}\right) & \text { for }(+\varepsilon) \\
L_{d}=2 A\left(d_{1} w_{3}\right) & \text { for }(-\varepsilon)
\end{array}
$$

\subsection{Temperature Distribution}

From appendix (A3):

$$
\begin{aligned}
& \frac{\Delta T}{\Delta \theta}=X \\
& T_{n}=T_{n-1}+X \Delta \theta \\
& T_{1}=T_{i}
\end{aligned}
$$

\subsection{Frictional Torque}

\subsubsection{The Case of $( \pm \varepsilon)$}

From appendix (A4.1):

$$
\begin{gathered}
M=M_{1}-M_{v} \\
M_{1}=\frac{1}{\varepsilon^{3}}\left[\frac{(H)^{2}}{2}-2(H)+\left(\alpha_{1} \beta_{1}+\sigma^{2}\right) \ln (H)+\frac{2 \sigma^{2}}{H}\right. \\
\left.-\frac{\alpha_{1} \beta_{1} \sigma^{2}}{2 H^{2}}\right]_{\theta_{i}}^{\theta_{o}} \\
M_{v}=\left(\frac{-K_{v}}{\varepsilon^{5}}\right)\left[\frac{H^{4}}{4}-\frac{4 H^{3}}{3}+\frac{\left(6+\sigma^{2}-2 \varepsilon^{2}\right) H^{2}}{2}\right. \\
-4\left(\alpha_{1} \beta_{1}+\sigma^{2}\right) H+\left(\alpha_{1}^{2} \beta_{1}^{2}+6 \sigma^{2}-2 \varepsilon^{2} \sigma^{2}\right) \ln (H) \\
\left.-\frac{4 \alpha_{1} \beta_{1} \sigma^{2}}{H}-\frac{\alpha_{1}^{2} \beta_{1}^{2} \sigma^{2}}{2 H^{2}}\right]_{\theta_{i}}^{\theta_{o}}
\end{gathered}
$$

\subsubsection{The First Exceptional Case $(\varepsilon=0)$} From appendix (A4.2):

$$
\begin{gathered}
M=M_{1}-M_{v}: \\
M_{1}=\left(1+\sigma^{2}\right)\left[\frac{\cos ^{3} \theta}{3}-\cos \theta\right]_{\theta_{i}}^{\theta_{o}} \\
M_{v}=\left(-K_{v}\right)\left(1+\sigma^{2}\right)\left[\cos \theta-\frac{2 \cos ^{3} \theta}{3}+\frac{\cos ^{5} \theta}{5}\right]_{\theta_{i}}^{\theta_{o}}
\end{gathered}
$$

\subsubsection{The Second Exceptional Case $(\varepsilon=1)$}

From appendix (A4.2):

$$
M=M_{1}-M_{v}
$$

$$
M_{1}=\left[\frac{H^{2}}{2}-2 H+\sigma^{2} \ln (H)+\frac{2 \sigma^{2}}{H}\right]_{\theta_{i}}^{\theta_{o}}
$$




$$
\begin{aligned}
M_{v}= & \left(-K_{v}\right)\left[\frac{H^{4}}{4}-\frac{4 H^{3}}{3}+\frac{\left(4+\sigma^{2}\right) H^{2}}{2}\right. \\
& \left.-4 \sigma^{2} H+4 \sigma^{2} \ln (H)\right]_{\theta_{i}}^{\theta_{o}}
\end{aligned}
$$

\subsection{Volume Flow Rate}

From Yacout [4]:

$$
Q= \pm A
$$

\subsection{Friction Factor}

From Yacout [1-4]:

$$
F=M / W
$$

\subsection{Stiffness Factor}

From Yacout [1-4]:

$$
S F=-(\bar{\beta} W+\beta \bar{W})
$$

\subsection{Bearing Losses}

\subsubsection{Pump Power}

From Yacout [1-4]:

$$
\begin{gathered}
p_{p}=q \cdot p_{s} \\
P_{p}=\left(\frac{\pi \beta p_{s}^{2} c^{3}}{6 \mu \varepsilon}\right) Q
\end{gathered}
$$

\subsubsection{Frictional Power}

Yacout [1-4]:

$$
\begin{gathered}
p_{f}=m_{o} \cdot \Omega \\
P_{f}=\left(\frac{2 \pi \mu R^{4} \Omega^{2}}{c}\right) M
\end{gathered}
$$

\subsubsection{Total Losses}

$$
P_{t}=P_{f}+P_{p}
$$

\section{Results}

Since the objective of this study is finding out the bearing thermal behavior under the lubricant viscosity variation in the presence of the centripetal inertia and the surface roughness, and because of the complexity of the theoretical solution specially for this type (clearance) it was necessary to select the viscosity form adopted by the author in a previous study but with minor deviation to prevent any approximation needed to derive the equations which can predict the bearing behavior. The bearing characteristics such as pressure distribution, temperature distribution, load carrying capacity; flow rate, frictional torque, friction factor and stiffness factor have been calculated for the bearing with hemispherical and partially hemispherical seats. Figures (2-49) represent the bearing with hemispherical and partially hemispherical seats.

\section{Discussion}

It is necessary to find out more about this bearing performance where the thermal behavior hasn't been accomplished in Yacout [4]. The visions, of Yacout [2-3] and others referenced in, of the lubricant viscosity and temperature gradient for the hydrostatic spherical bearing have been adopted. The designed bearing example offered in Yacout [4] has been checked as a guide in the present study.

\subsection{The Pressure Distribution}

Unlike the fitted type the clearance one has a broad characteristic spectrum and different special cases. Trying to cover this bearing performance with least possible figures without loosing any significant information, six cases in addition to the two special ones have been studied.

Figures (2-17) represent the effects of the different parameters on the pressure distribution for a bearing with hemispherical and partial hemispherical seats.
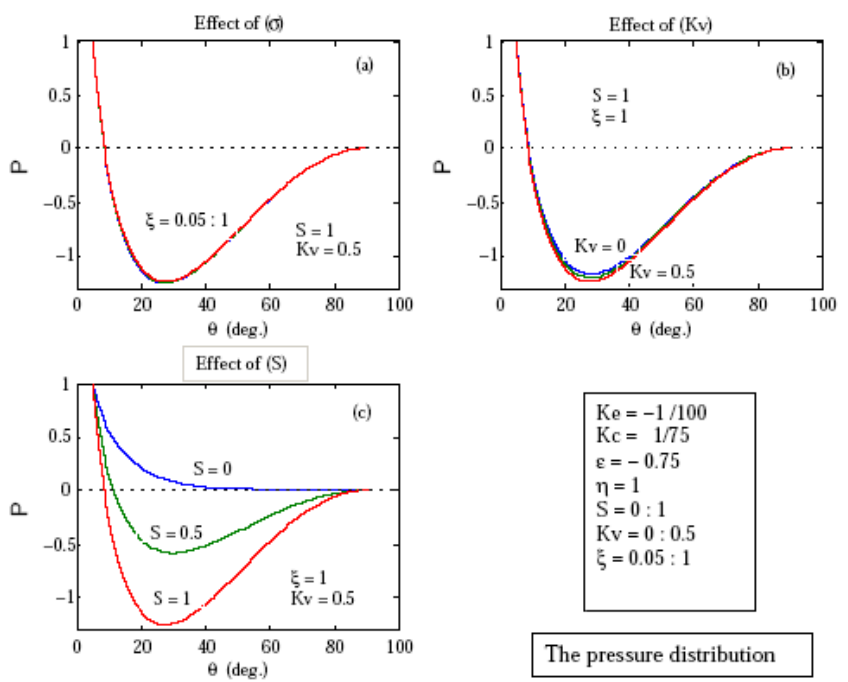

Figure 2. Hemispherical seat.
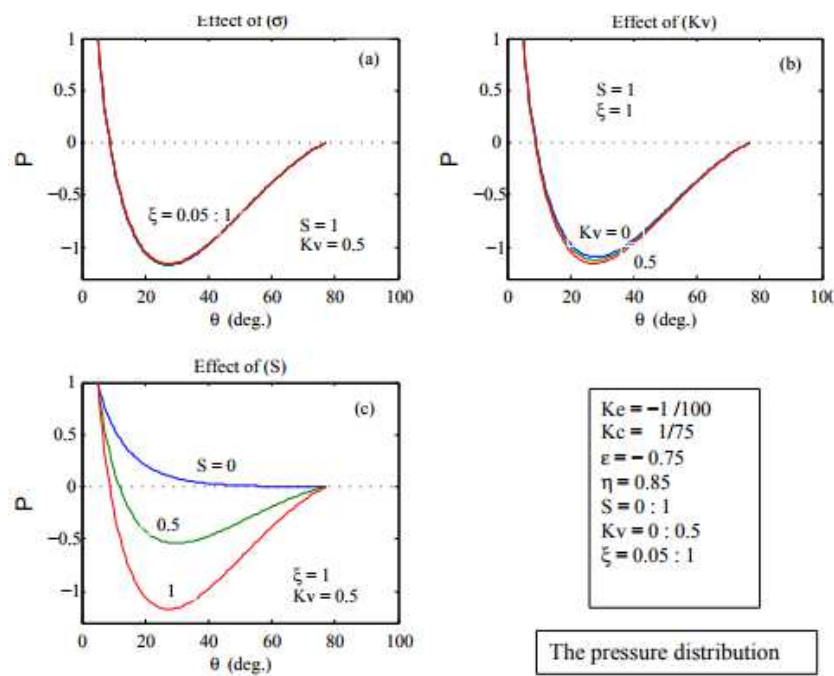

The pressure distribution

Figure 3. Partial hemispherical seat. 

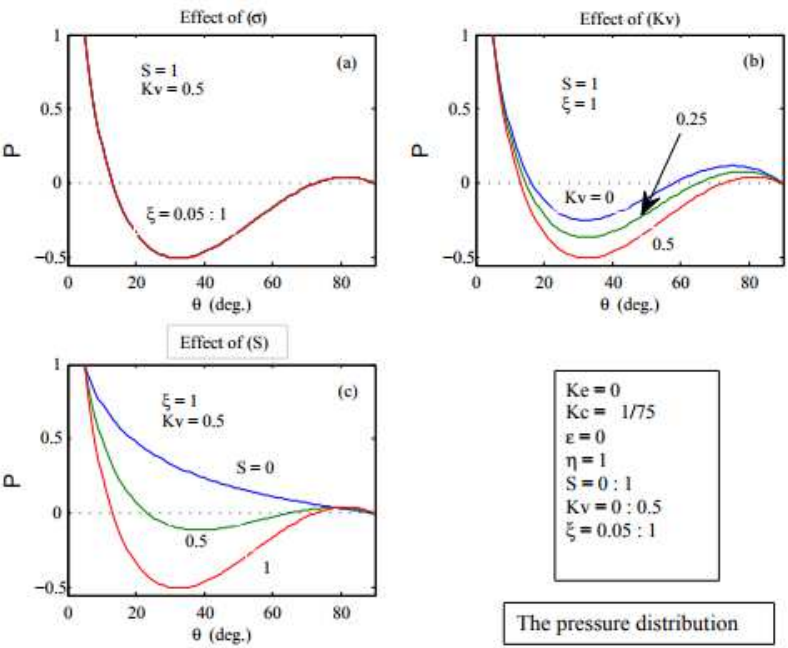

Figure 4. Hemispherical seat.
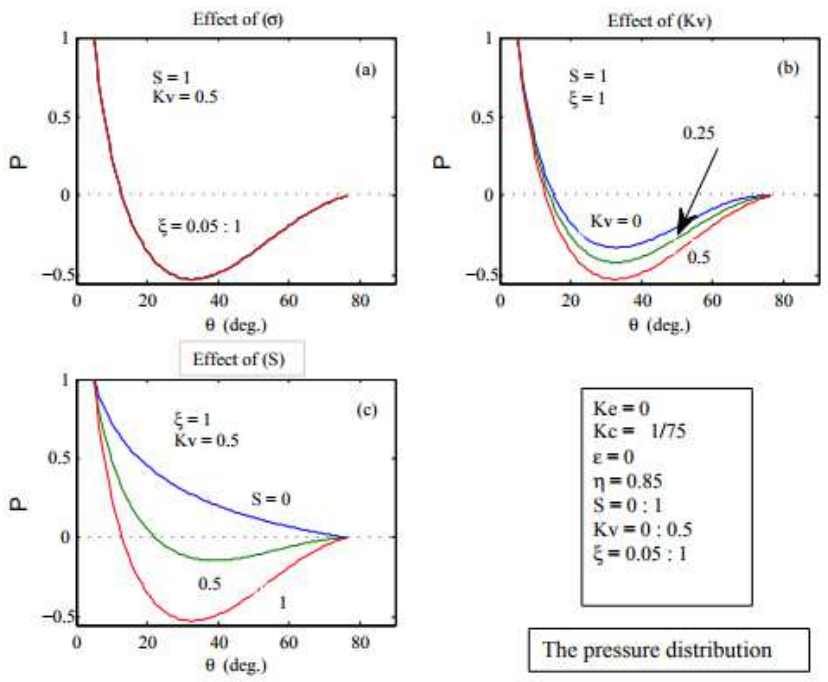

Figure 5. Partial hemispherical seat.
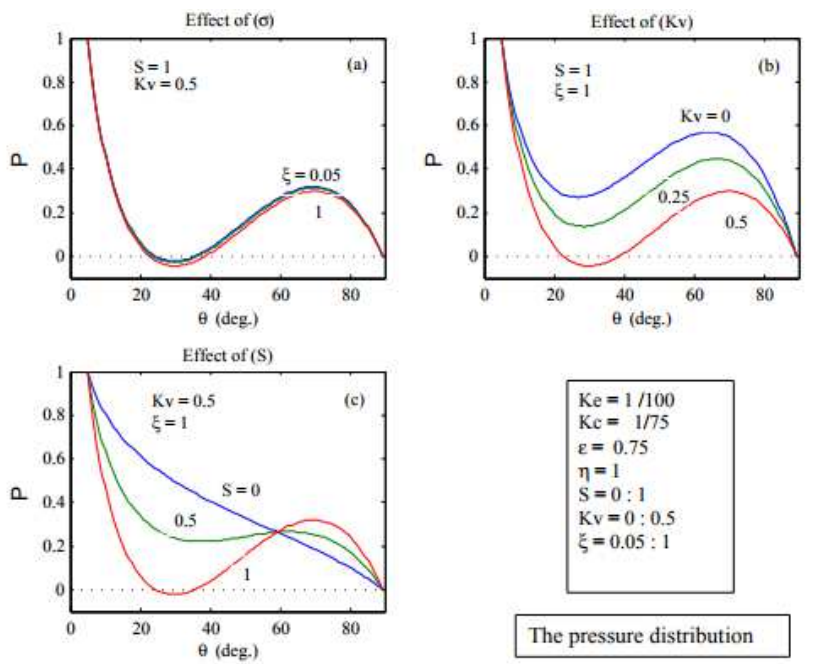

Figure 6. Hemispherical seat.
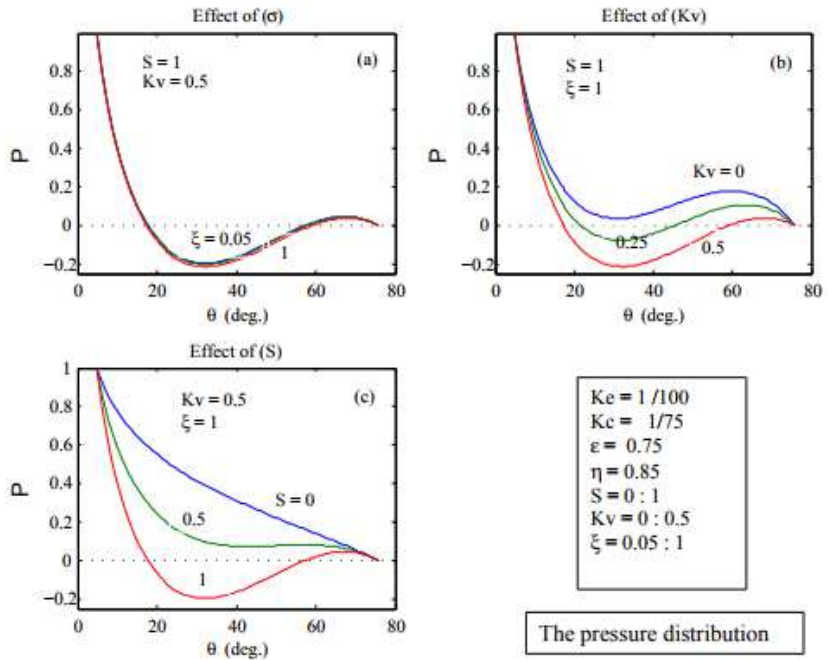

$\mathrm{Ke}=1 / 100$ $K c=1 / 7$
$\varepsilon=0.75$ $\varepsilon=0.75$
$\eta=0.85$

$\mathrm{S}=0: 1$

$\mathrm{Kv}=0: 0.5$

$\xi=0.05: 1$

The pressure distribution

Figure 7. Partial hemispherical seat.
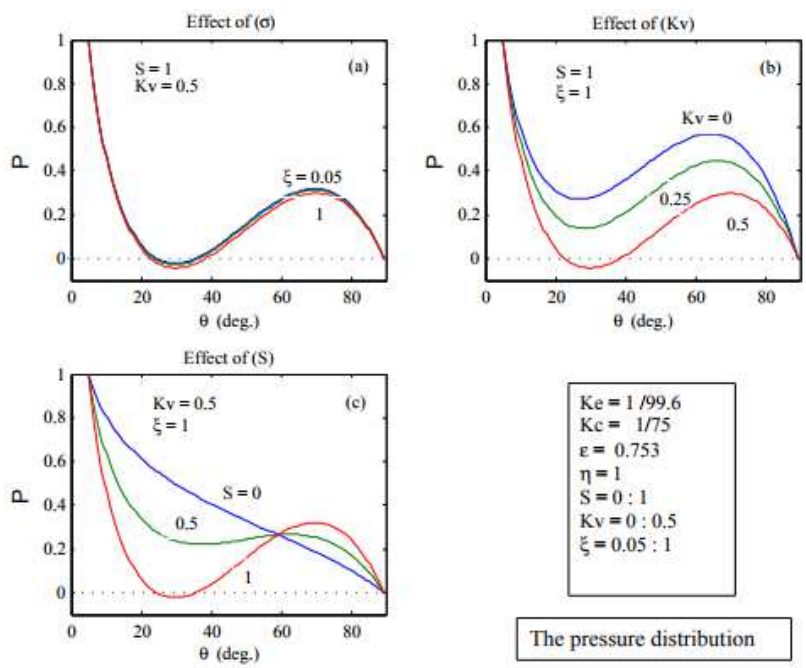

Figure 8. Hemispherical seat.
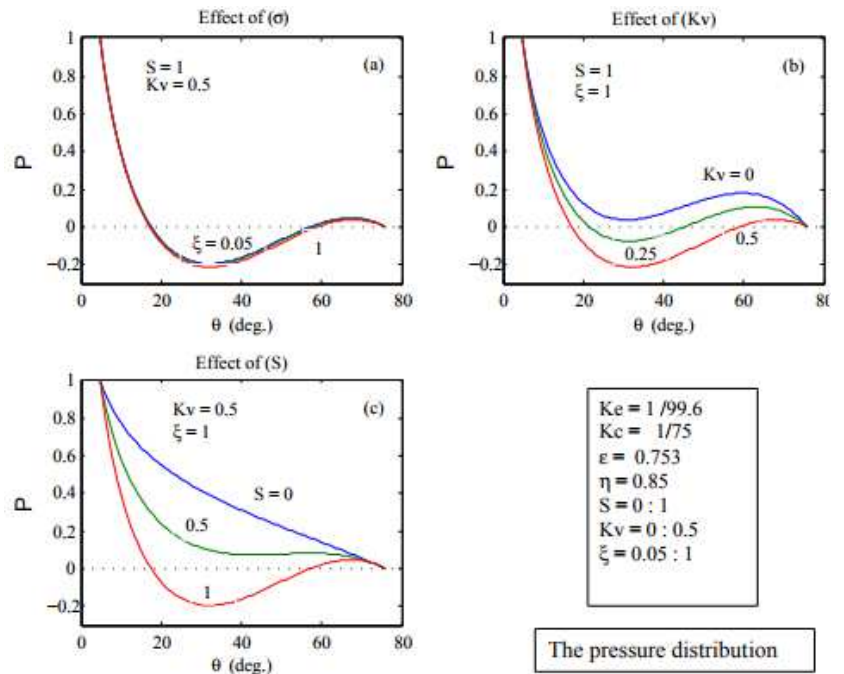

Figure 9. Partial hemispherical seat. 

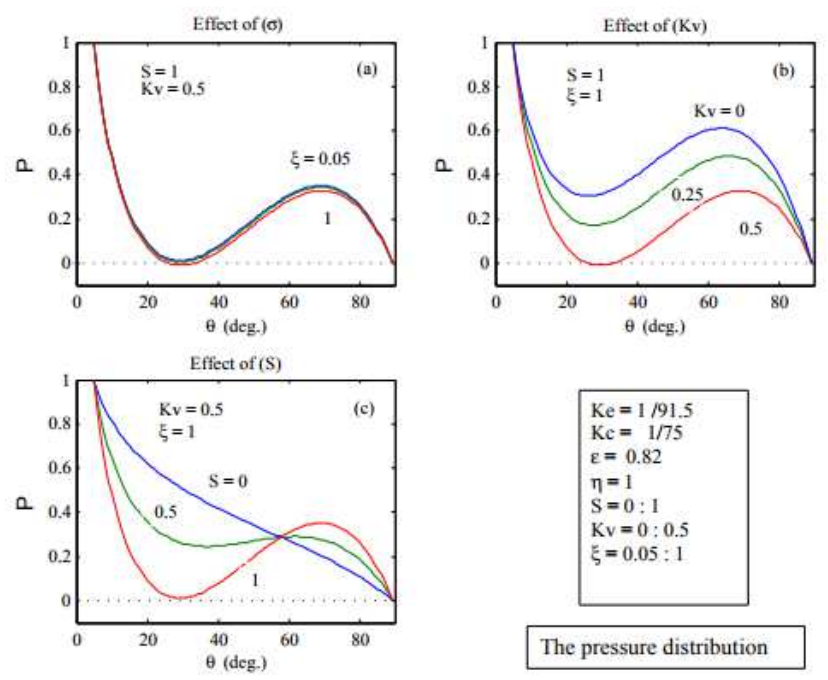

Figure 10. Hemispherical seat.
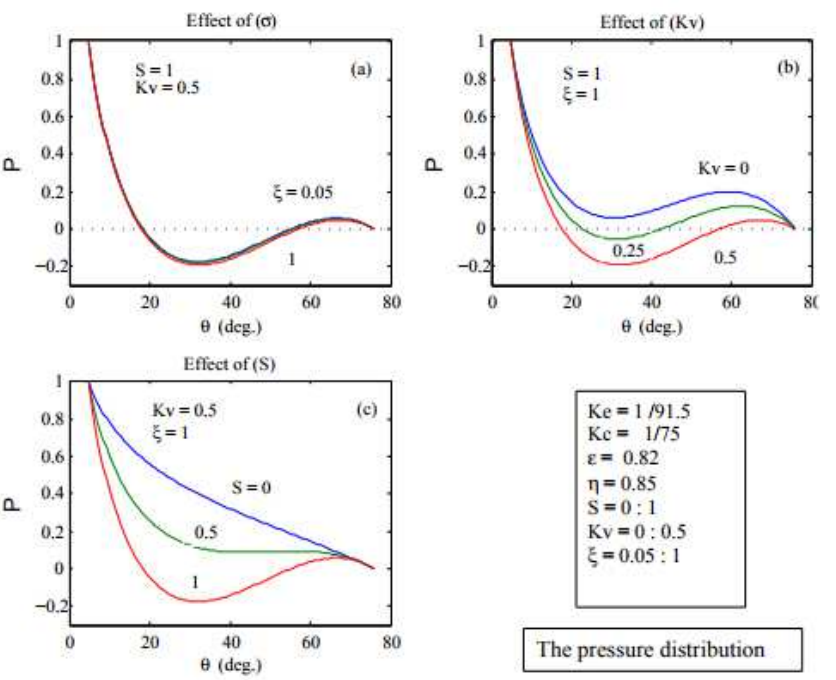

Figure 11. Partial hemispherical seat.
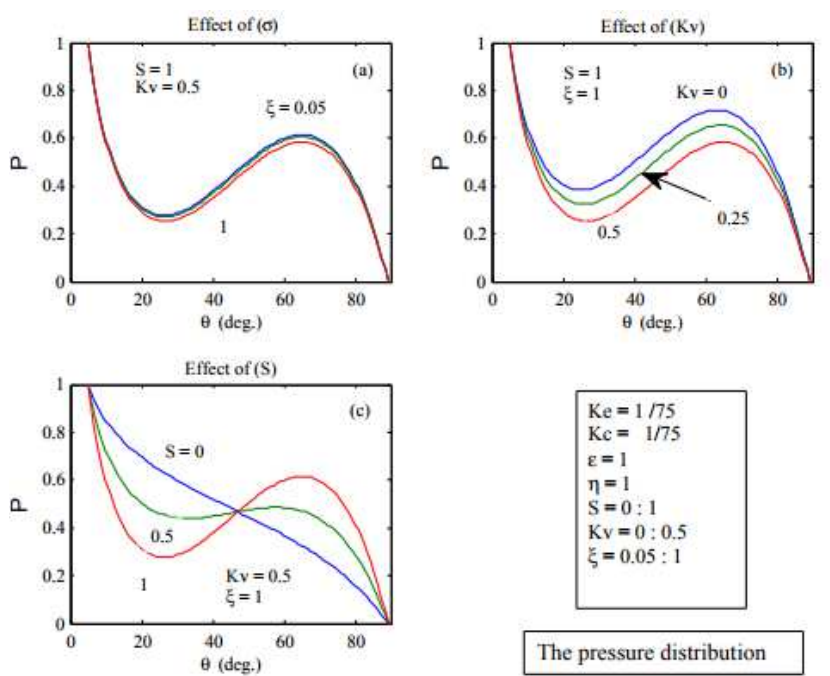

Figure 12. Hemispherical seat.
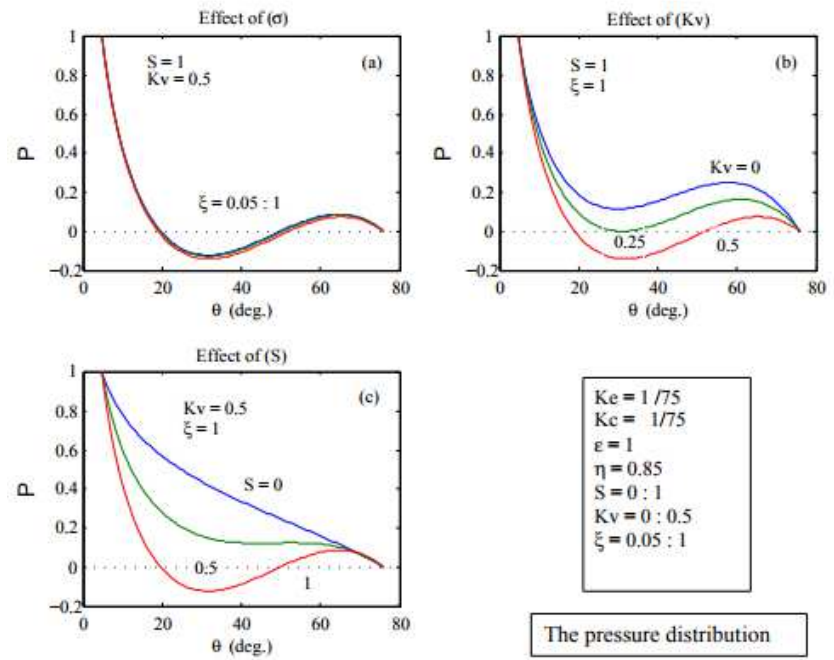

Figure 13. Partial hemispherical seat.
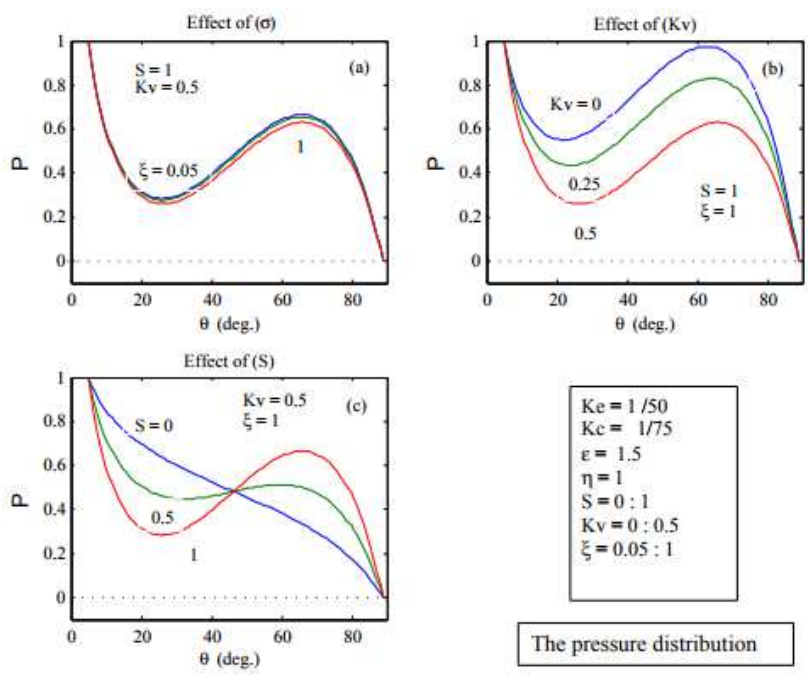

Figure 14. Hemispherical seat.
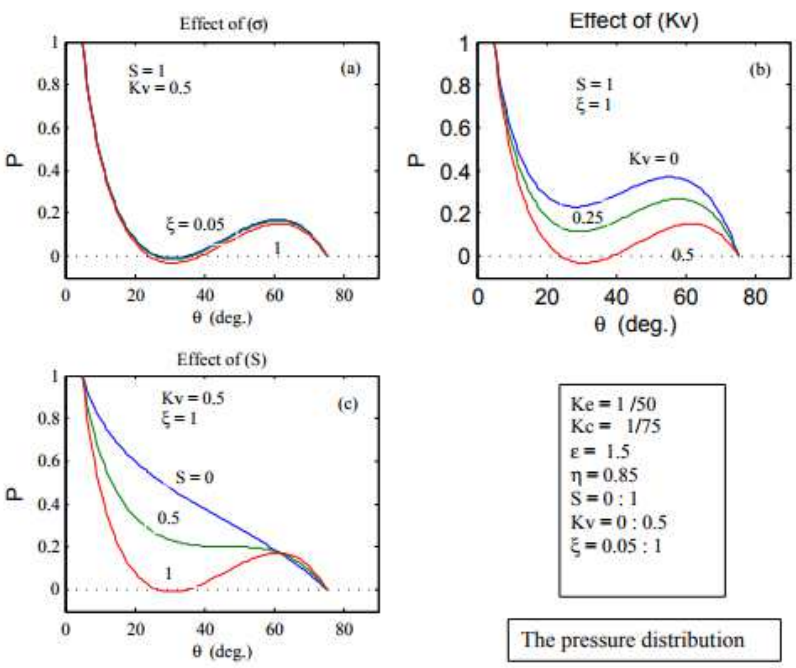

Figure 15. Partial hemispherical seat. 

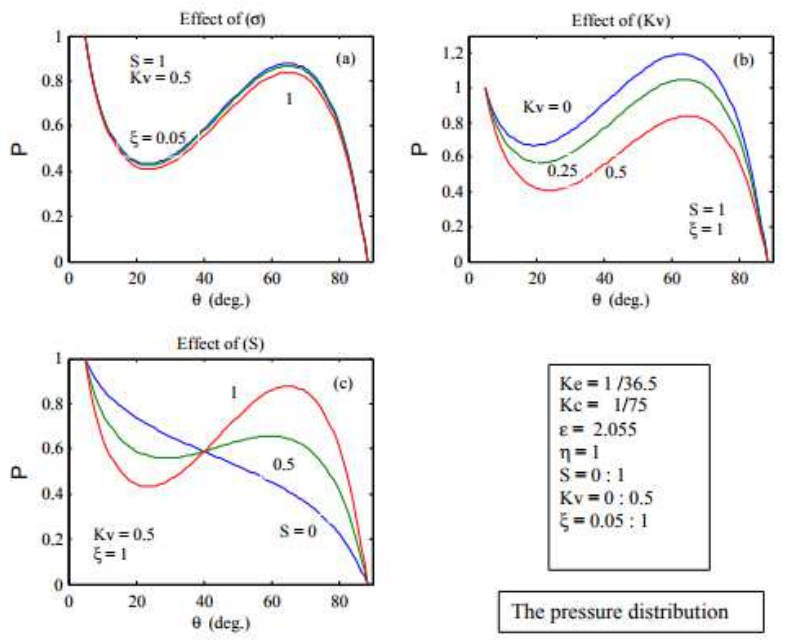

Figure 16. Hemispherical seat.
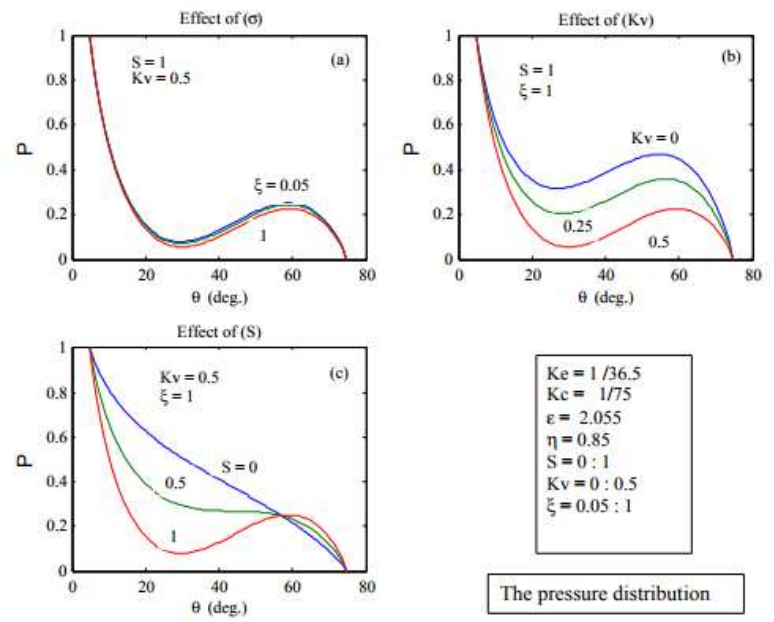

Figure 17. Partial hemispherical seat.

\subsubsection{The Effect of the Surface Roughness}

Subfigures (2a-17a) show that the pressure in the case of both bearing configurations, hemispherical and Partial hemispherical, hasn't been significantly affected by the surface roughness. Compared with the results in Yacout [4] no remarkable difference could be observed despite the viscosity variation.

\subsubsection{The Effect of the Viscosity Variation}

The viscosity effect on the pressure could be easily observed where Subfigures (2b-17b) show inverse relation between the viscosity, represented by the viscosity variation constant, and the pressure. Because of this inverse relation attention should be given to cases of high speeds (high inertia) to avoid negative pressures.

\subsubsection{The Effect of the Inertia}

As in Yacout [1-4] the inertia represented by the speed parameter has a dominant effect on the pressure where it can highly increase or decrease the pressure depending on the eccentricity ratios. It can lead to negative pressures especially at low and negative eccentricity ratios of both bearing configurations, subfigures $(2 \mathrm{c}-17 \mathrm{c})$.
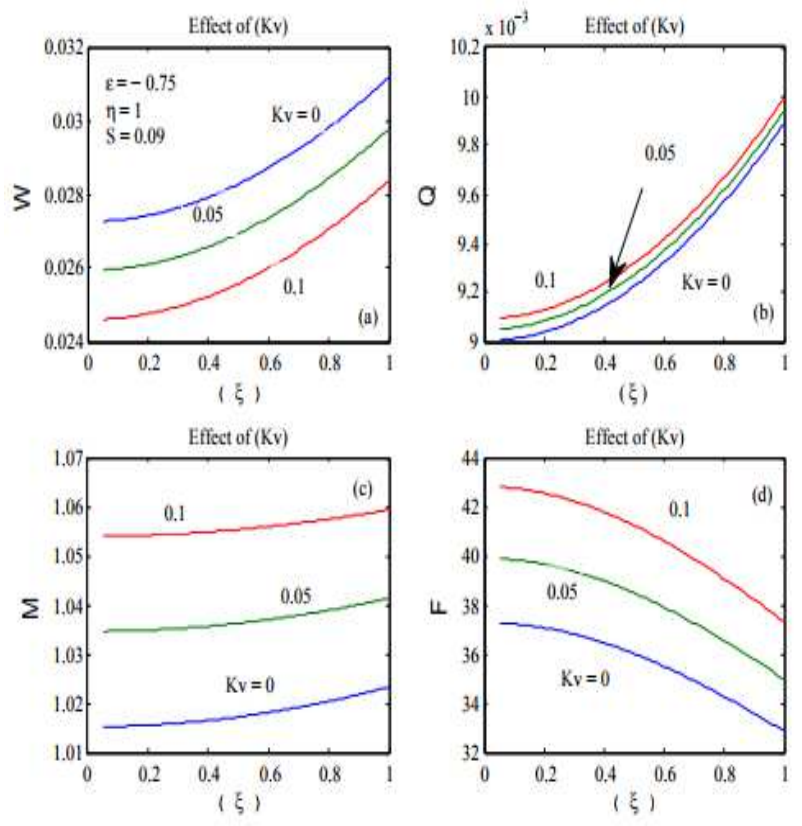

Figure 18. Hemispherical seat.
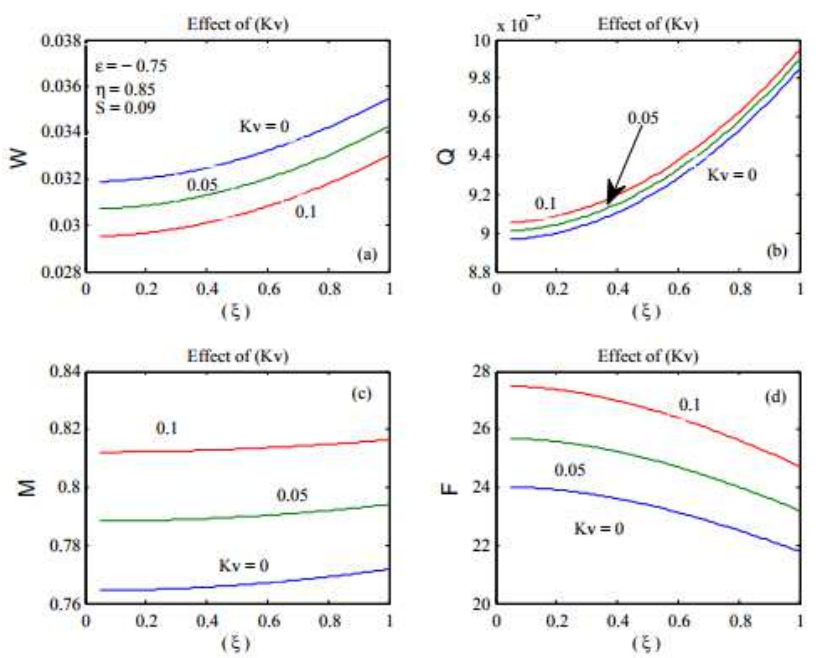

Figure 19. Partial hemispherical seat.
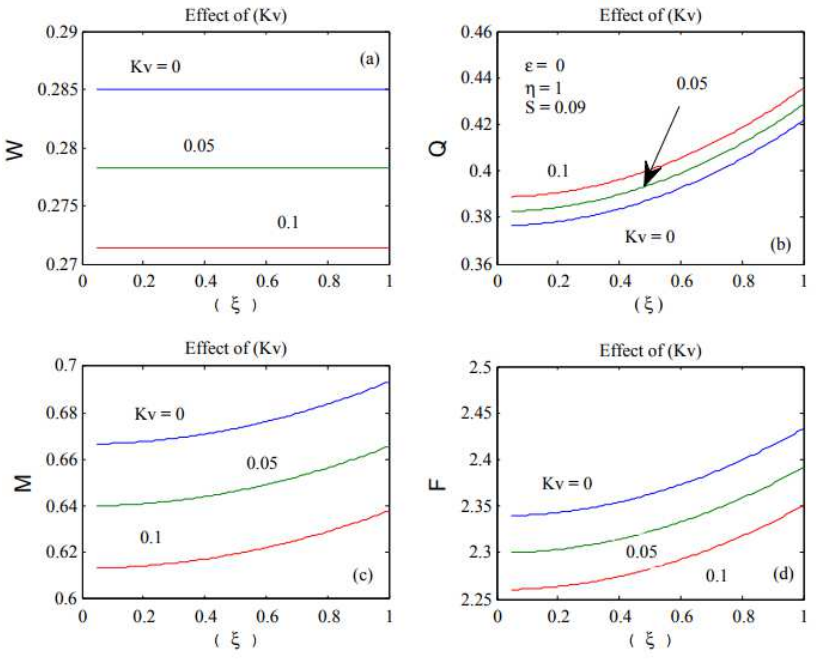

Figure 20. Hemispherical seat. 

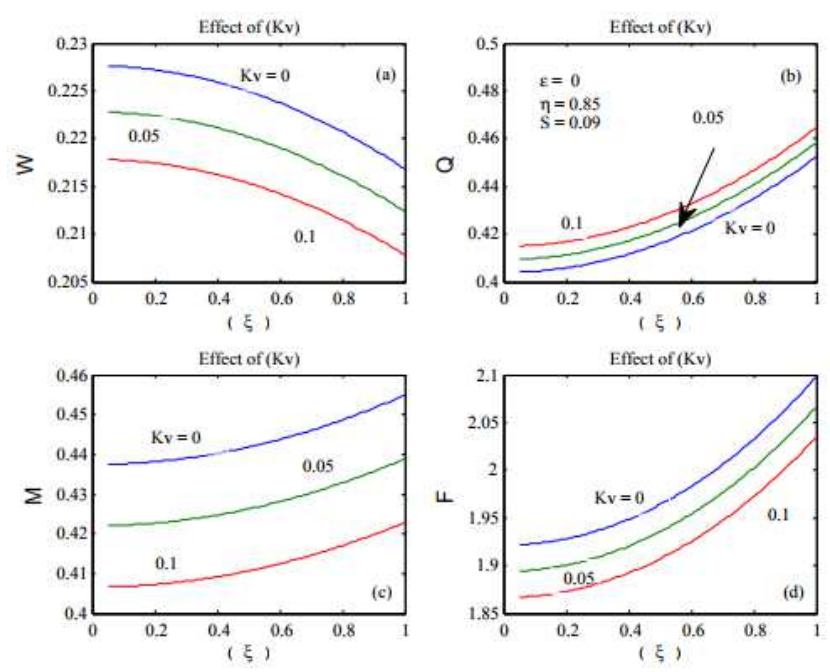

Figure 21. Partial hemispherical seat.
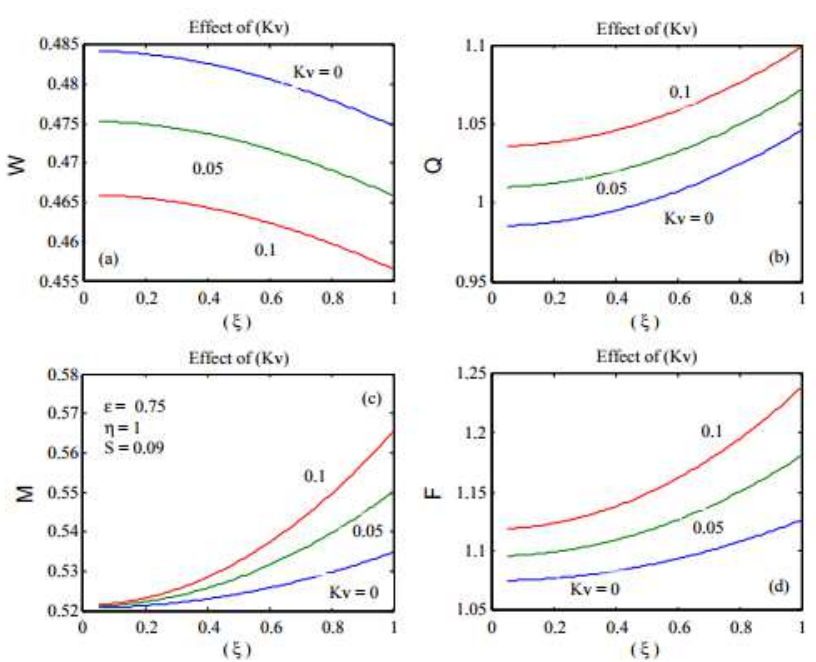

Figure 22. Hemispherical seat.
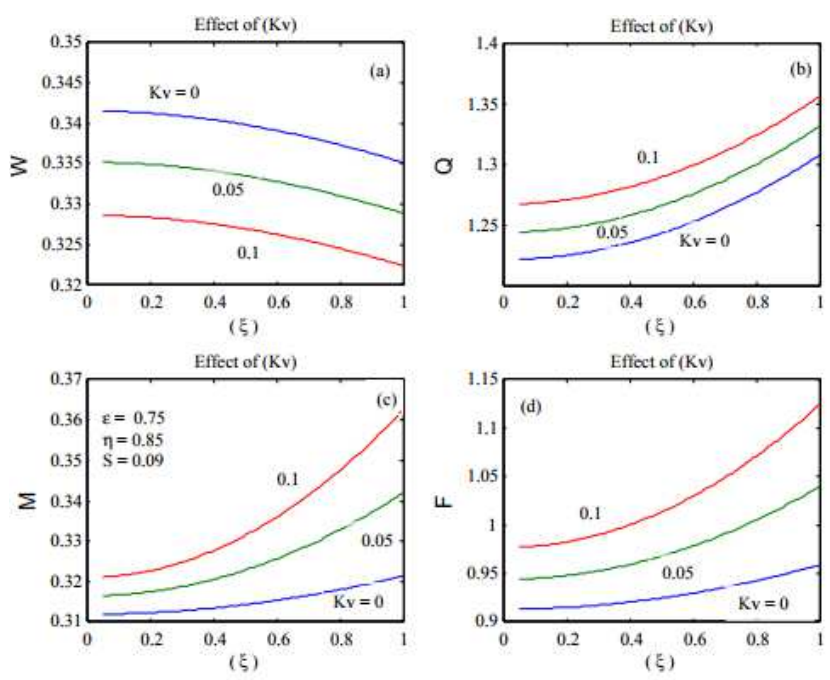

Figure 23. Partial hemispherical seat.
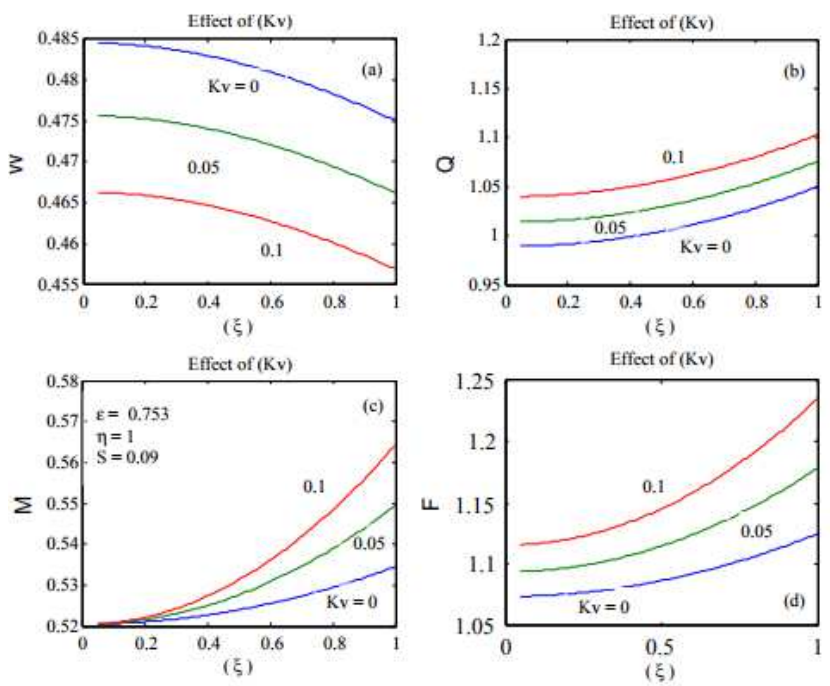

Figure 24. Hemispherical seat.
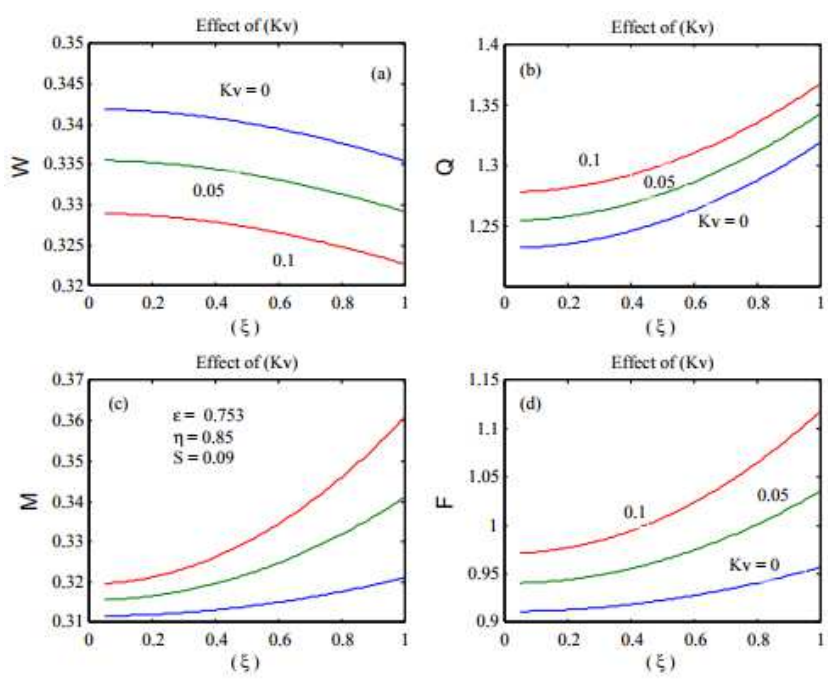

Figure 25. Partial hemispherical seat.
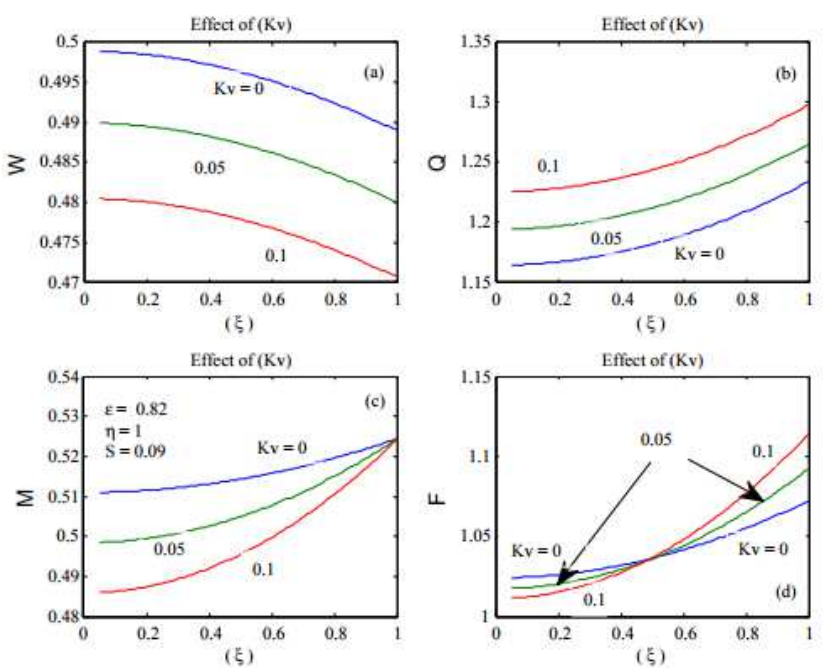

Figure 26. Hemispherical seat. 

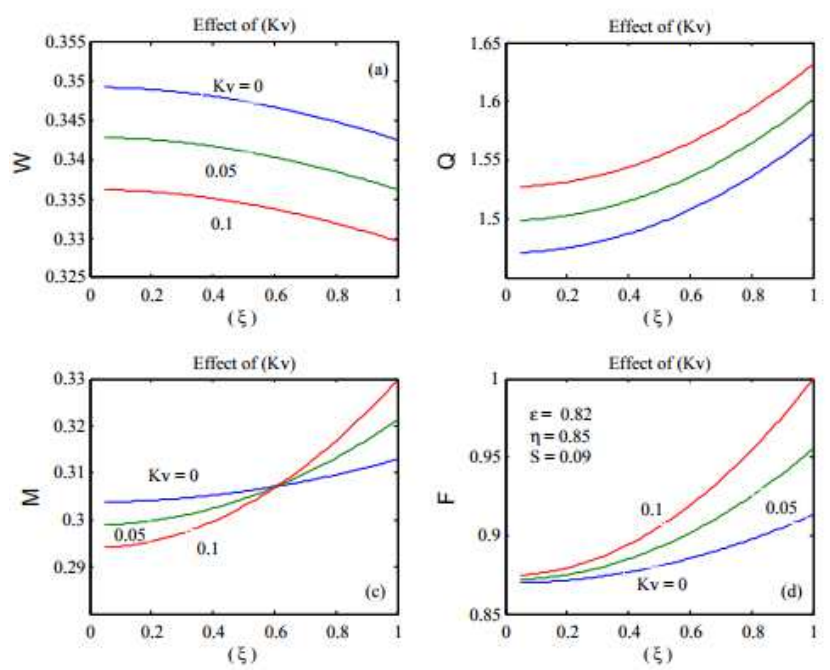

Figure 27. Partial hemispherical seat.
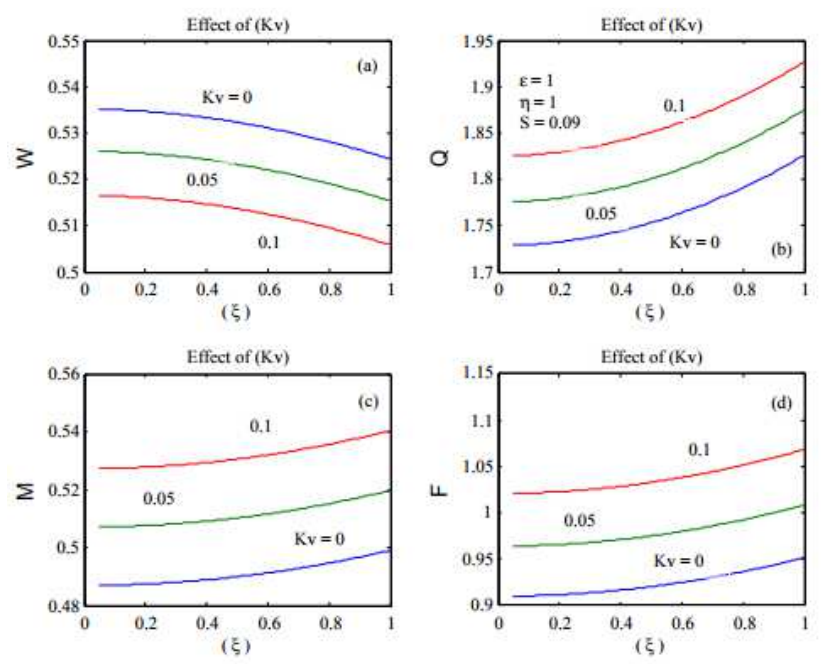

Figure 28. Hemispherical seat.
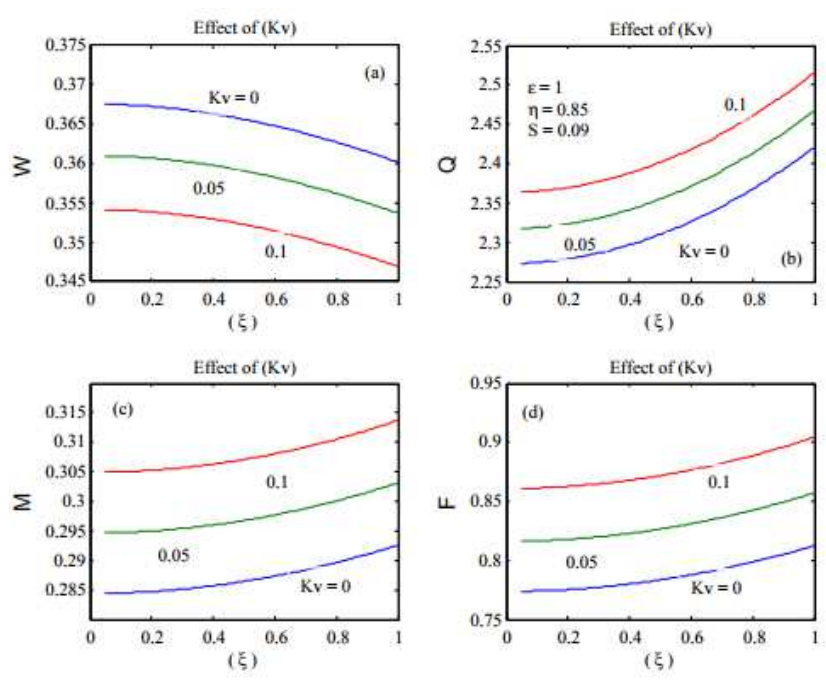

Figure 29. Partial hemispherical seat.
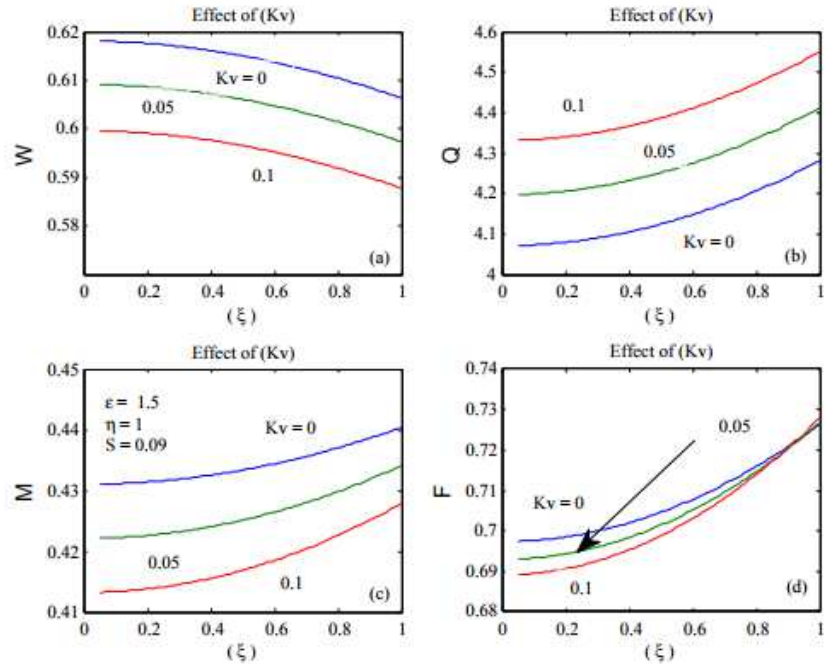

Figure 30. Hemispherical seat.
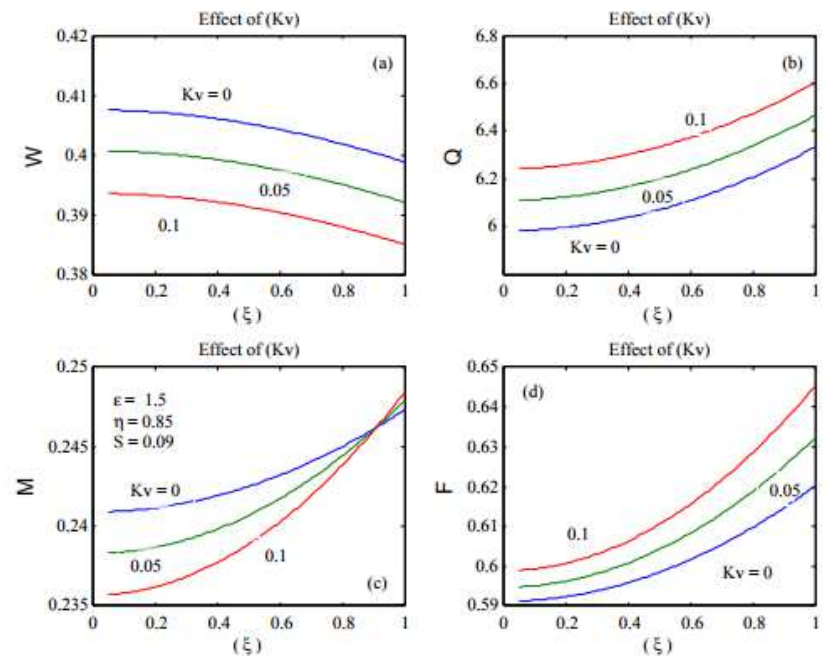

Figure 31. Partial hemispherical seat.
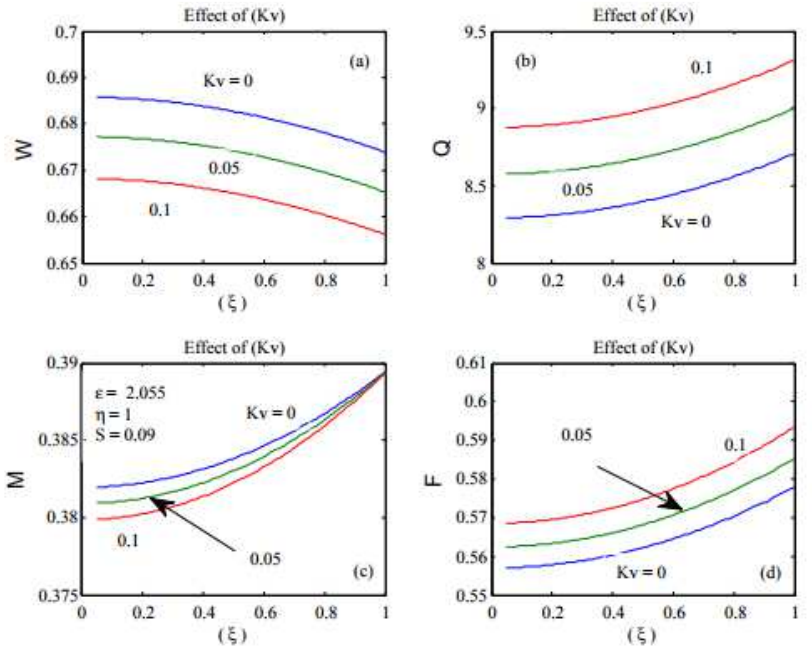

Figure 32. Hemispherical seat. 

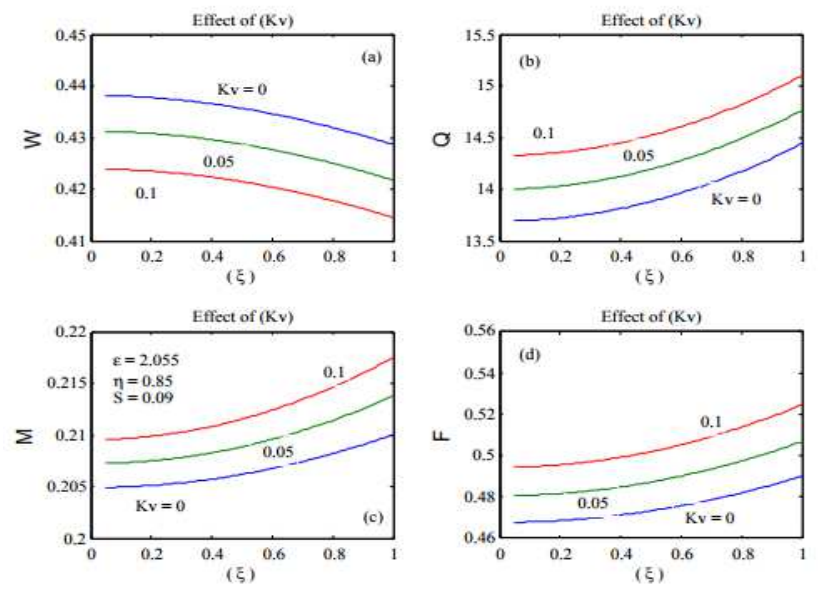

Figure 33. Partial hemispherical seat.

\subsection{The Load Carrying Capacity}

Subfigures (18a-33a) represent the effects of the variable viscosity and the surface roughness on the load carrying capacity at low speed parameter (low inertia) and different eccentricity ratios. The behavior complexity of this type of bearings could be obviously observed.

\subsubsection{The Effect of the Viscosity Variation}

The load decreases with the increase in the viscosity variation constant at the length of the eccentricity ratio spectrum, subfigures (18a-33a).

\subsubsection{The Effect of the Surface Roughness}

The load decreases with the increase insurface roughness at the positive eccentricity ratios while increases at the negative ones subfigure (18a-19a).

\subsubsection{The Effect of the Bearing Configuration}

At zero eccentricity ratio the load of the hemispherical configuration remains constant with the surface roughness increase while the load of the partial hemispherical one decreases, subfigures (20a-21a).

\subsection{The Lubricant Flow Rate}

Subfigures (18b-33b) show that the increase in the viscosity variation constant as well as the surface roughness leads to increase the lubricant flow rate at any eccentricity ratios.

\subsection{The Fictional Torque}

Subfigures (18c-33c) obviously show bearing behavior complexity where the frictional torque increases or decreases depending on the complicated relationship between the surface roughness, the viscosity, the eccentricity ratio and the bearing configuration; hence the eight studied cases have been carefully selected.

\subsubsection{The Effect of the Surface Roughness}

The aforementioned Subfigures show the frictional torque increase with the increase in the surface roughness without exceptions.

\subsubsection{The Effect of the Viscosity Variation}

The dominant factor controlling the frictional torque is the viscosity variation constant where it leads to increase or decrease the frictional torque depending on eccentricity ratio and the bearing configuration.

\subsection{The Fiction Factor}

Represented by subfigures (18d-33d) the friction factor assure the bearing behavior complexity it (friction factor), also as the frictional torque, increases or decreases depending on the complicated relationship between the surface roughness, the viscosity, the eccentricity ratio and the bearing configuration. Like the case of the frictional torque, the surface roughness increases the friction factor without exceptions while the viscosity variation constant increases or decreases the friction factor depending on eccentricity ratio and the bearing configuration.

\subsection{The Stiffness Factor}

Figures (34-35) represent the stiffness of a bearing (with different restrictors), which is determined numerically based on infinitesimally exciting (changing) the eccentricity and relating the infinitesimal excitation of the load to the infinitesimal excitation of the eccentricity through the infinitesimal excitation of the central pressure ratio as seen in appendix (A.5); it means that this bearing stiffness is in the thrust direction as in Yacout [1-4].

\subsubsection{The Effect of the Surface Roughness}

The aforementioned figures (34-35) show that the stiffness is greatly improved by surface roughness while the central pressure is decreased.

\subsubsection{The Effect of the Viscosity Variation}
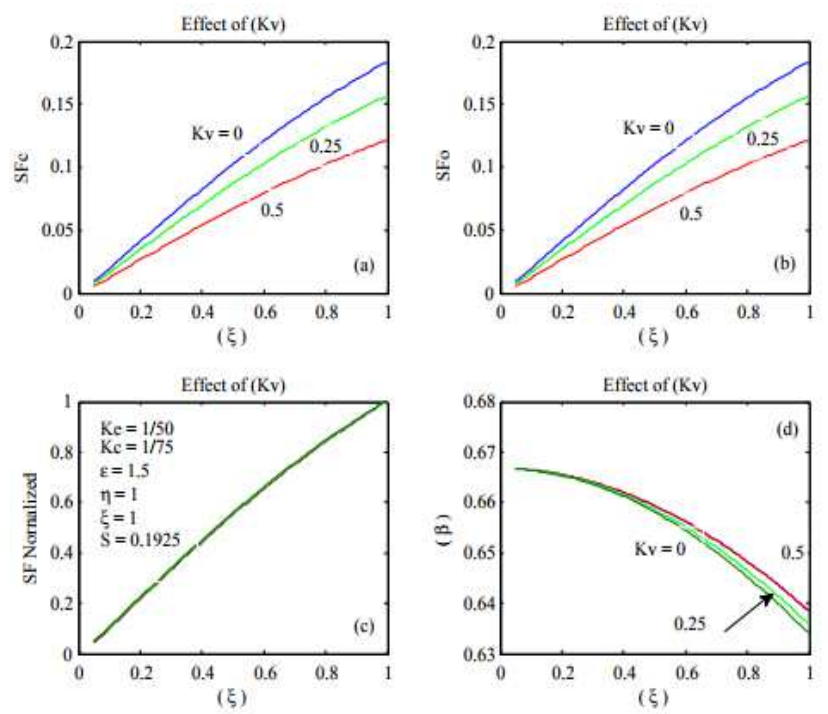

Figure 34. Hemispherical seat. 

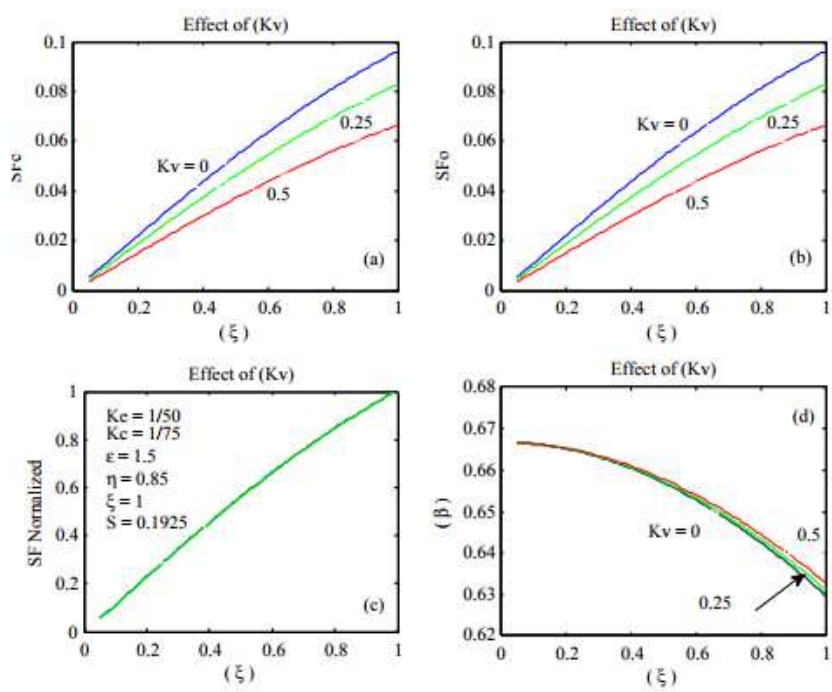

Figure 35. Partial hemispherical seat.

Subfigures (34a-b \& 35a-b) show that the stiffness is negatively affected by viscosity variation while the central pressure is slightly positively affected in the presence of high surface roughness subfigures (34d-35d), regardless the restrictor type. Normalizing the stiffness factor, subfigures $(34 c-35 c)$ show that the bearing performance hasn't been affected by the viscosity variation while it hasn't been greatly affected by the surface roughness.

\subsection{The Temperature Rise and Distribution}

The temperature rise is numerically calculated as in Yacout (2-3) using the initial temperature as zero degrees centigrade to make the temperature distribution curves express the temperature rise distribution figures (36-37).
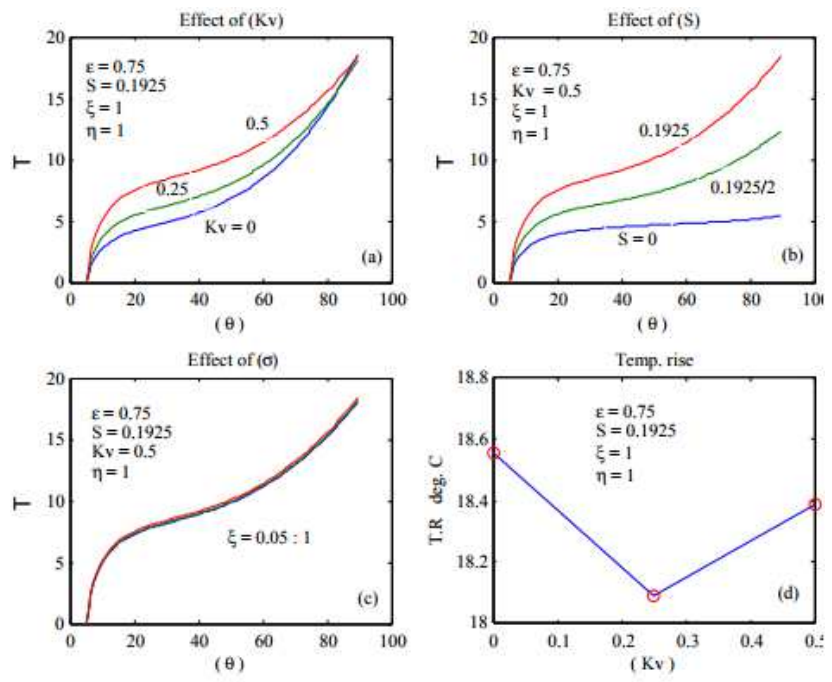

Figure 36. Hemispherical seat.

\subsubsection{The Effect of the Viscosity Variation}

Subfigures (36a-37a). Show the viscosity variation effect on the temperature rise distribution where the local rise increases with the increase in the viscosity variation constant except at the exit zone of the hemispherical seat subfigures (36d-37d).

\subsubsection{The Effect of the Centripetal Inertia}

Subfigures (36b-37b) show that the inertia severely increases the local temperature rise.

\subsubsection{The Effect of the Surface Roughness}

Subfigures (36c-37c) show insensible effect of the surface roughness on the temperature rise.
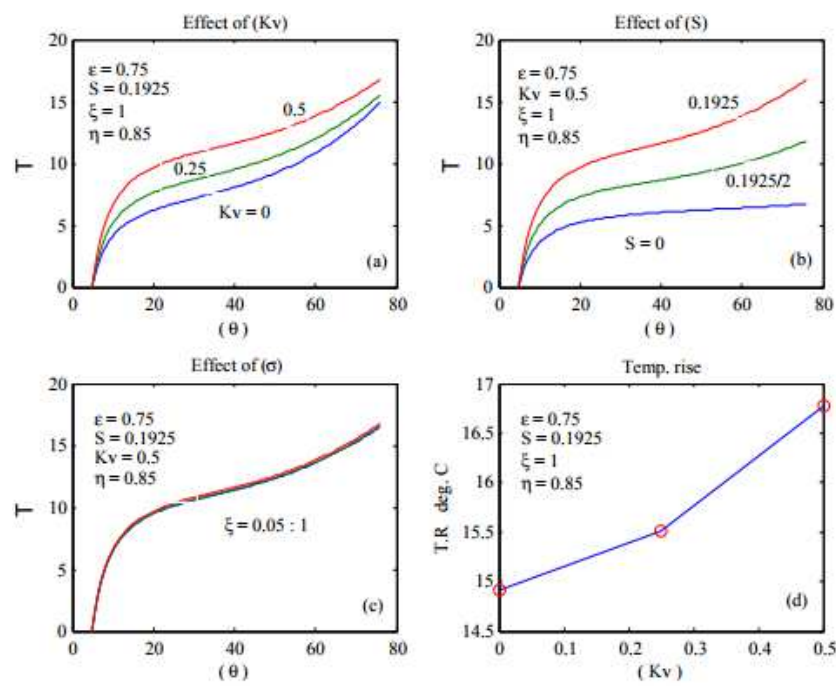

Figure 37. Partial hemispherical seat.

\subsection{The Natural Dynamic Phenomenon}

Looking at the pressure equation, it could be directly realized that it includes two logarithmic terms with negative values depending on the eccentricity ratio appendix (A1.4). This case has been overcome, in Yacout [4], through finding two forms for the same equation ignoring completely the physical meaning of such case. In the present study, these two logarithmic terms have been simply treated through varying their forms mathematically to keep the pressure equation in one form only avoiding the complications in Yacout [4]. It should be revealed that solving the problem of the logarithmic negative values doesn't mean preventing the dynamic pressure in turn the dynamic load. This 'Natural Dynamic Phenomenon' disappears in the two special cases of such bearing where it is related to the positive and negative eccentricity ratios only. Because of the importance and the novelty of this natural dynamic phenomenon which needs to be deeply investigated, the present study will just touch the case postponing the deep study to a future separate paper figures (38-45).

\subsubsection{The Dynamic Pressure}

Subfigures (38a-b-45a-b) show that the dynamic pressure with its two parts, real and imaginary, increases in the exit direction regardless the sign of the eccentricity ratio except the real part in case of positive eccentricity ratios where it decreases. The viscosity variation has insensible effect on the real part while increases the imaginary part.

\subsubsection{The Dynamic Load}

Subfigures (38c-d-45c-d) show that the viscosity variation and the surface roughness increase both parts of the dynamic 
load regardless the sign of the eccentricity ratio without any exceptions.
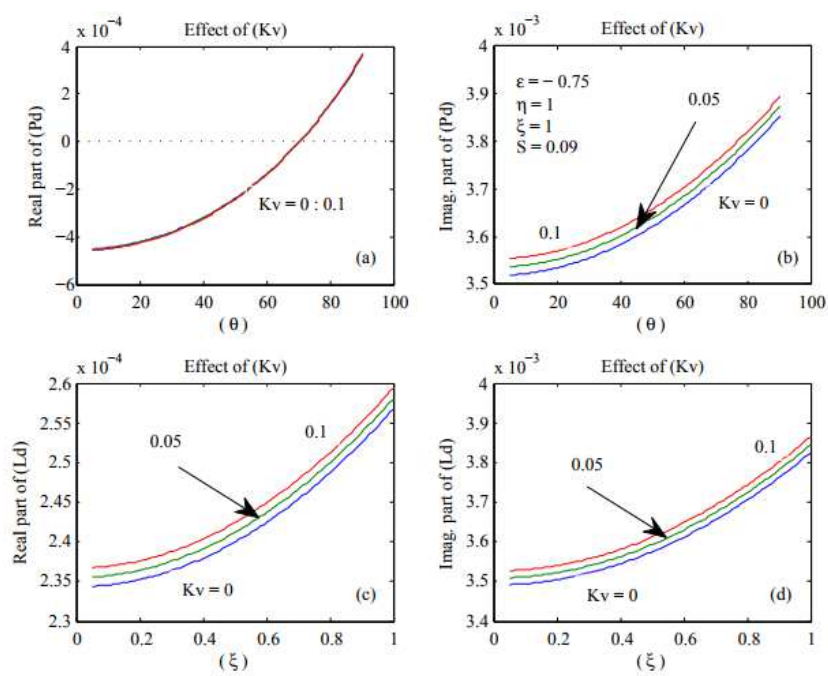

Figure 38. Hemispherical seat.
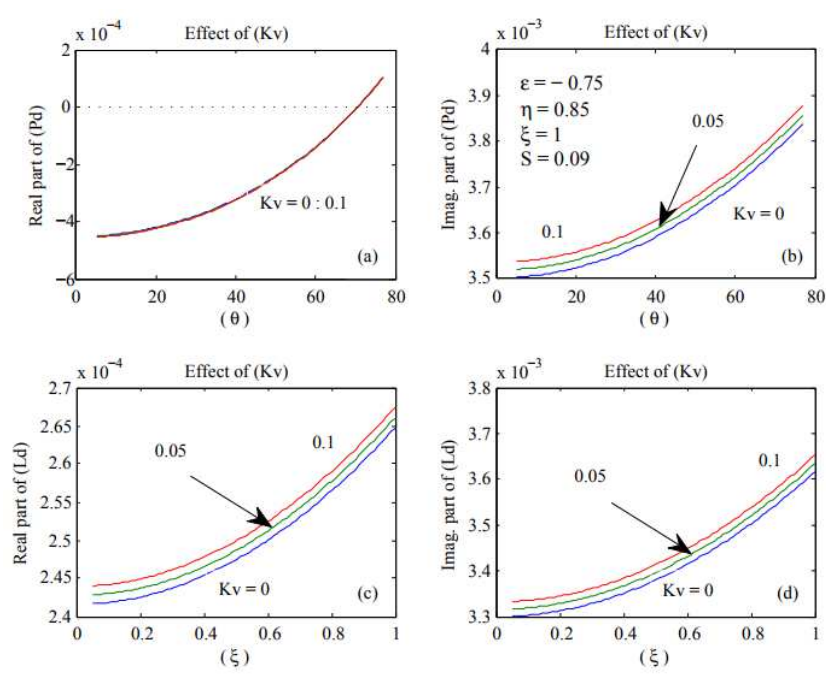

Figure 39. Partial hemispherical seat.
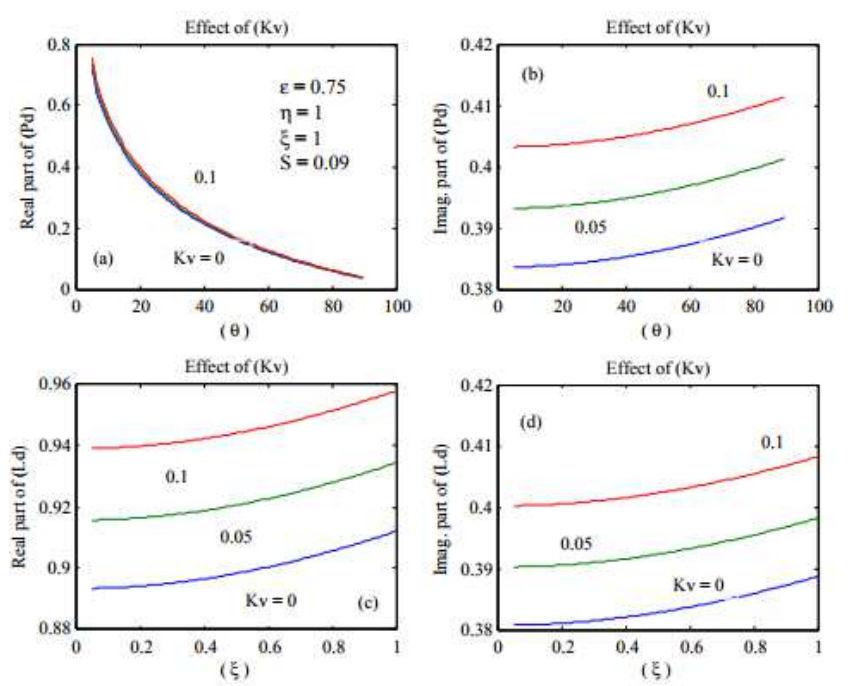
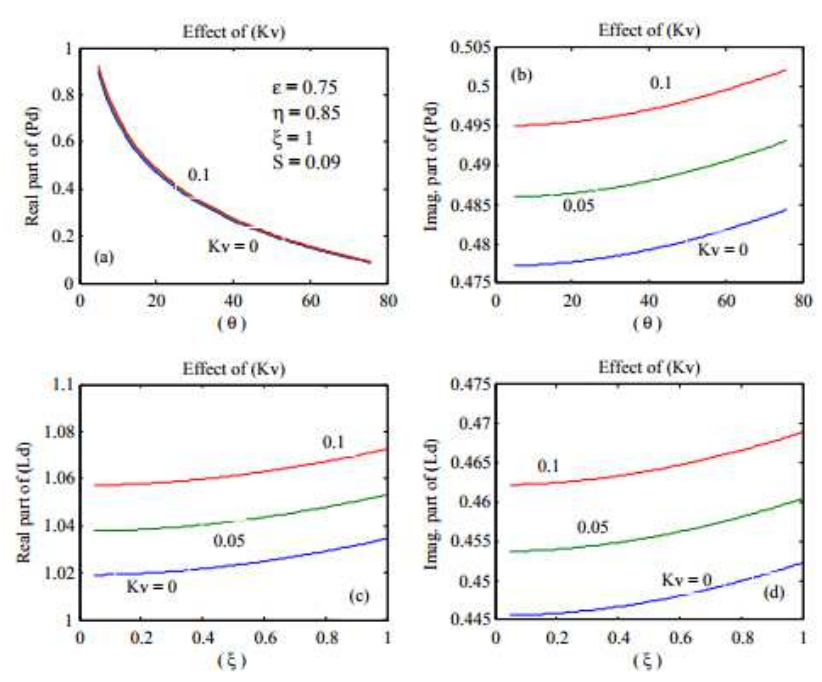

Figure 41. Partial hemispherical seat.
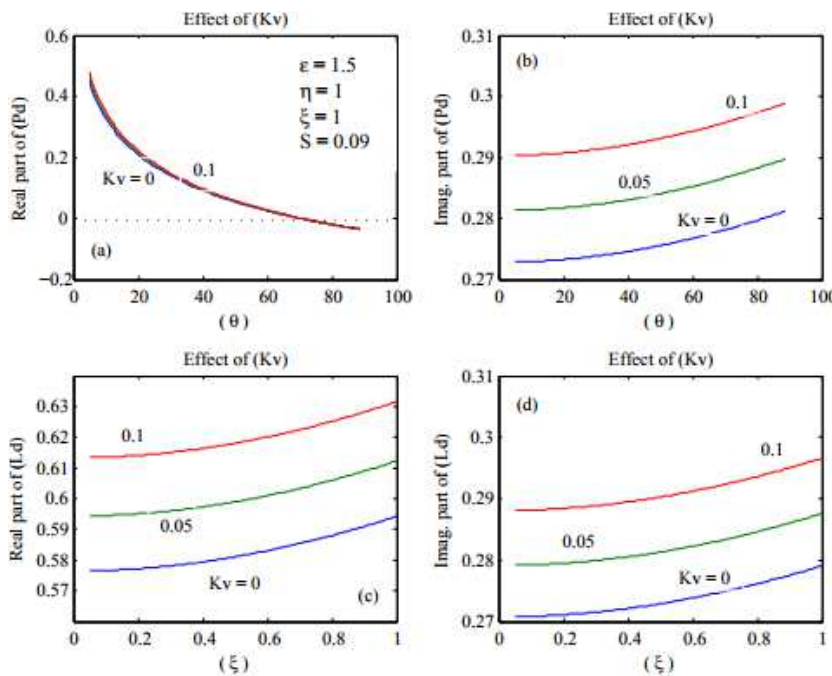

Figure 42. Hemispherical seat.
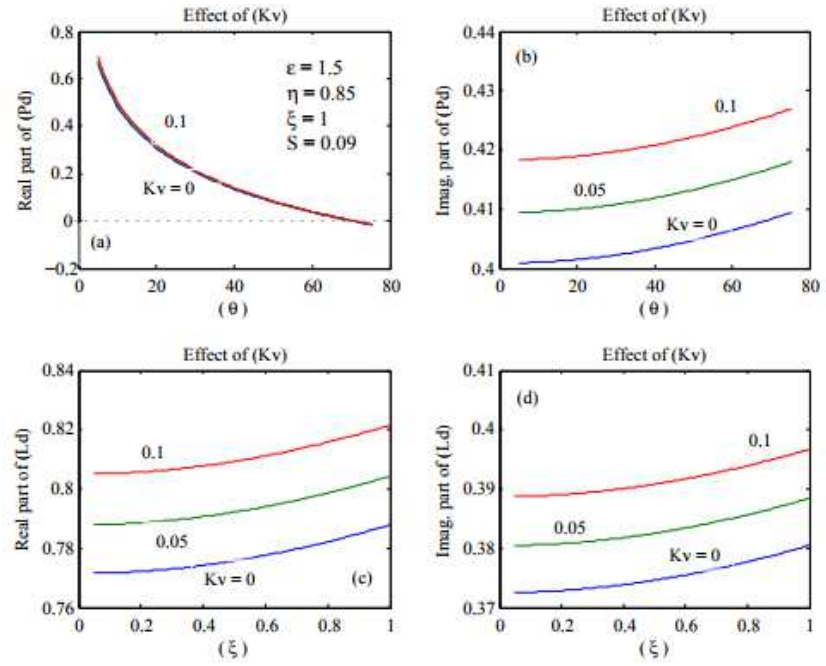

Figure 43. Partial hemispherical seat.

Figure 40. Hemispherical seat. 

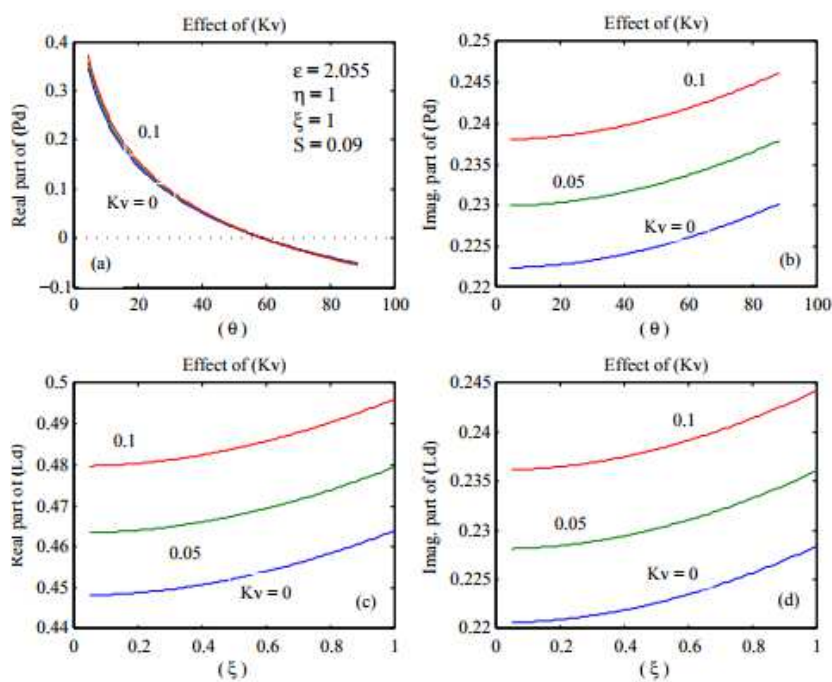

Figure 44. Hemispherical seat.
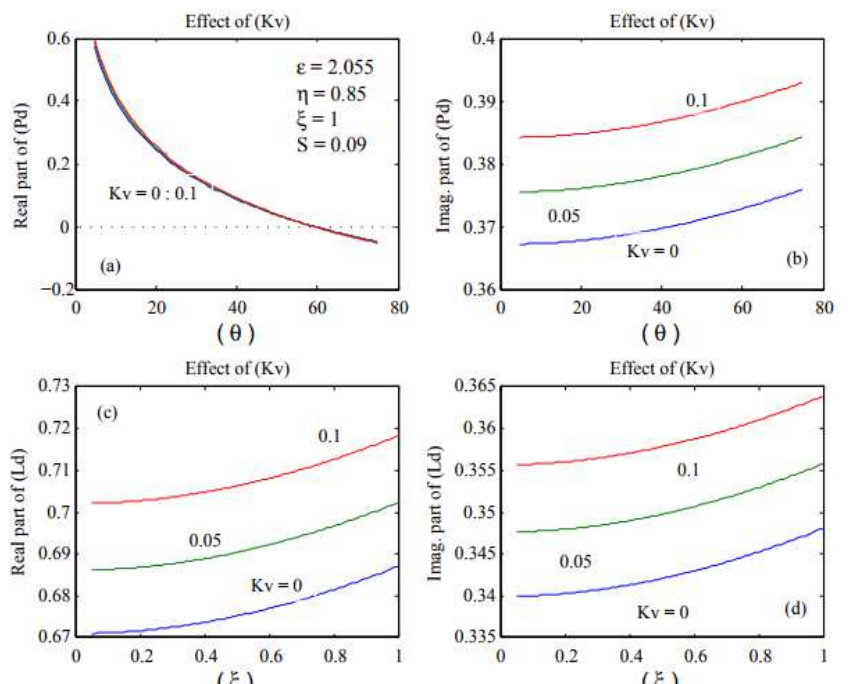

Figure 45. Partial hemispherical seat.
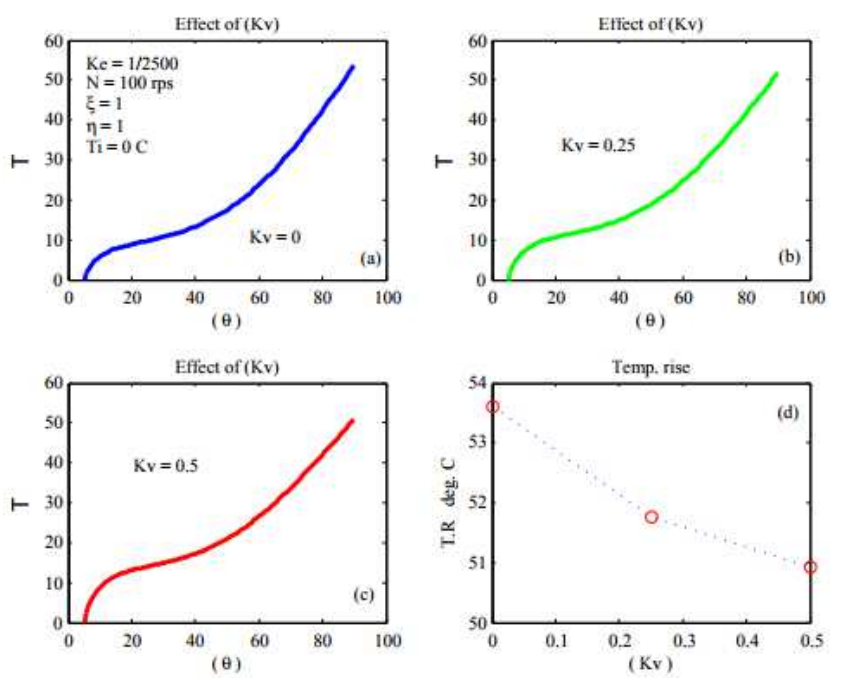

Figure 46. Hemispherical seat.
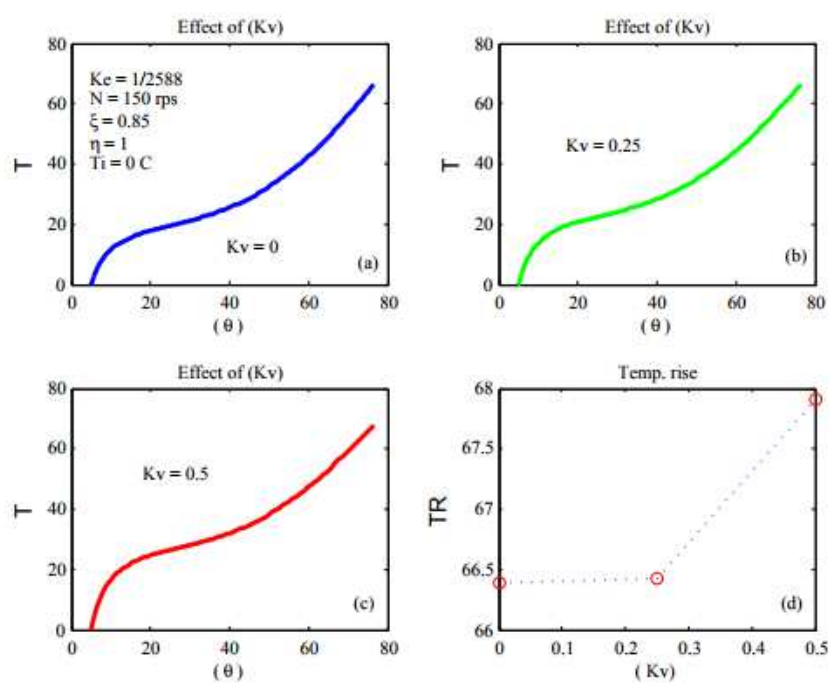

Figure 47. Partial hemispherical seat.
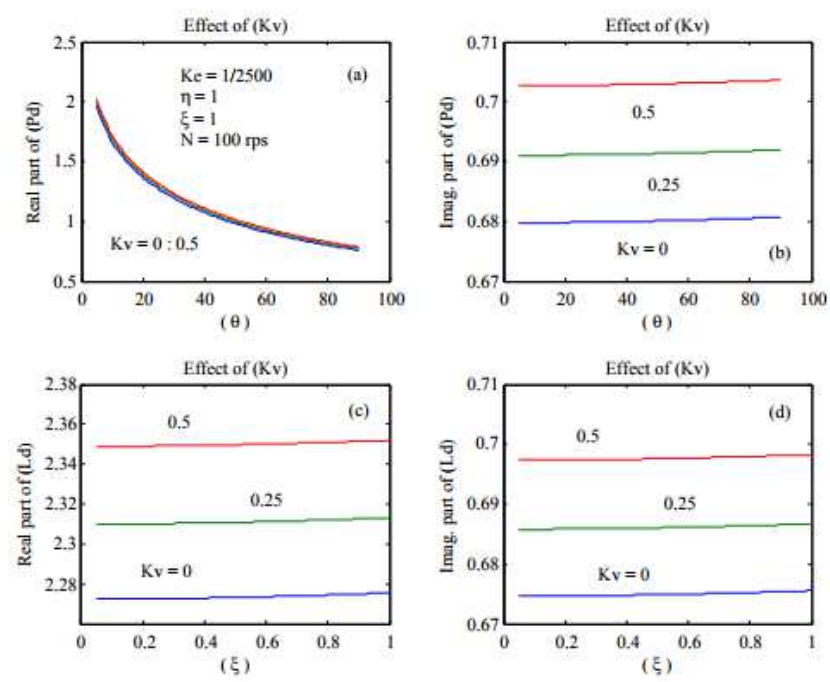

Figure 48. Hemispherical seat.
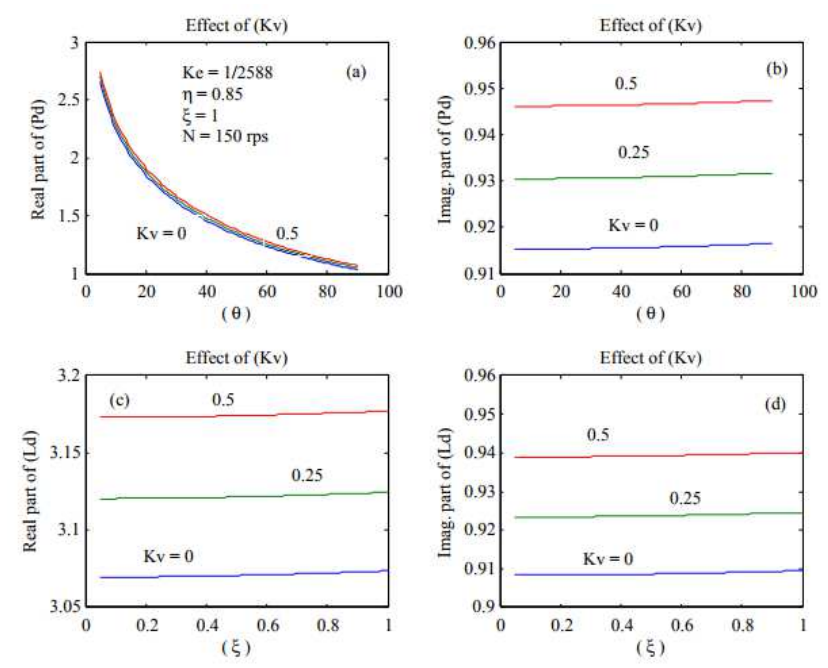

Figure 49. Partial hemispherical seat.

\subsection{Checking the Design Example}

Yacut [4], offered an example for an un-recessed clearance 
bearing designed based on the minimum power losses, minimum flow rate and optimal restrictor dimensions.

\subsubsection{Checking the Bearing Temperature Rise}

Checking the bearing reveals its high temperature rise which reaches to be around sixty degrees centigrade for both configurations. While slightly decreases the temperature rise of the hemispherical configuration the viscosity variation increases the temperature rise of the other configuration. figures (46-47).

\subsubsection{Checking the Bearing Dynamic Phenomenon}

Figures (48-49) show that the bearing dynamic pressure and load are very high for both configurations.

\section{Conclusion}

From the above mathematical calculations, figures and discussion, this type of bearings could be really called a mysterious bearing; hence it is believed that this study is just a step on the way to correctly design this type of bearings. However, the aforementioned discussion could be concluded as:

1. The pressure equation is mathematically reformed to be put only in one form avoiding the difficulties in the previous study.

2. The high inertia and high viscosity variation constant lead to negative pressures and loads.

3. The surface roughness plays a dominant role in bearing stiffness.

4. The bearing dynamic phenomenon should be carefully investigated.

5. The previous design technique, based on minimum power losses, minimum flow rate and optimal restrictor dimensions proved its invalidity.

6. This type of bearings should be redesigned, after the careful study of its dynamic phenomenon, based on more factors.

\section{Future Work}

The next work will investigate and study the dynamic phenomenon of this bearing in details to find out the nature of this force and how it affects the bearing performance.

\section{Appendix}

\section{Appendix 1. Integration of the Pressure Equation}

\section{Appendix 1.1. Pressure Distribution $( \pm \varepsilon)$}

From Yacout [1-4]:

$$
\begin{gathered}
h_{o}=c+e \cos \theta \\
\frac{d p}{d \theta}=\frac{C}{\left(h_{o}^{3}+3 \sigma_{o}^{2} h_{o}\right) \sin \theta}+\frac{3}{20} \rho \Omega^{2} R^{2} \sin 2 \theta
\end{gathered}
$$

Through introducing the non-dimensional quantities:

$$
\begin{gathered}
P=\frac{p}{p_{i}}, H=\frac{h_{o}}{c}, S=\frac{3}{40} \frac{\rho \Omega^{2} R^{2}}{p_{i}} \\
\sigma=\frac{\sigma_{o}}{c}, C=\frac{-6 \mu q}{\pi}, b^{2}=3 \sigma^{2}, \\
\mu=\mu_{i}\left(1-K_{v} \sin ^{2} \theta\right), a_{c}=\frac{-6 \mu_{i} q}{\pi c^{3} p_{i}}
\end{gathered}
$$

Equation (1) becomes:

$$
\begin{aligned}
& \frac{d P}{d \theta}=a_{c}\left(\frac{1-K_{v} \sin ^{2} \theta}{\left(H^{3}+b^{2} H\right) \sin \theta}\right)+2 S \sin (2 \theta) \\
& d P=\left[\left(\frac{a_{c}}{\left(H^{3}+b^{2} H\right) \sin \theta}\right)+2 S \sin (2 \theta)\right] d \theta \\
& \quad-a_{c}\left(\frac{K_{v} \sin ^{2} \theta}{\left(H^{3}+b^{2} H\right) \sin \theta}\right) d \theta \\
& d P=d P_{1}-d P_{v} \\
& \int d P=\int d P_{1}-\int d P_{v} \\
& P=P_{1}-P_{v}
\end{aligned}
$$

Where:

$$
\begin{aligned}
& d P_{1}=\left[\left(\frac{a_{c}}{\left(H^{3}+b^{2} H\right) \sin \theta}\right)+2 S \sin (2 \theta)\right] d \theta \\
& d P_{v}=\left(\frac{a_{c} K_{v} \sin ^{2} \theta}{\left(H^{3}+b^{2} H\right) \sin \theta}\right) d \theta
\end{aligned}
$$

The integration of ( $\left.d P_{1}\right)$ could be easily found in Yacout [4] or in another form as:

$$
\begin{aligned}
P_{1}= & A\left[a_{1} \ln (H)+\frac{c_{1}}{2} \ln \left(H-\alpha_{1}\right)^{2}\right. \\
& \left.+\frac{d_{1}}{2} \ln \left(H-\beta_{1}\right)^{2}+\frac{e_{1}}{2} \ln \left(H^{2}+b^{2}\right\}+\frac{f_{1}}{b} \tan ^{-1}\left(\frac{H}{b}\right)\right] \\
& -S \cos (2 \theta)+B
\end{aligned}
$$

The integration of $\left(d P_{v}\right)$ is:

$$
P_{v}=\left(\frac{A K_{v}}{8 \varepsilon^{6}}\right)\left[a_{1 v} \ln (H)+\frac{e_{1 v}}{2} \ln \left(H^{2}+b^{2}\right)+\frac{f_{1 v}}{b} \tan ^{-1}\left(\frac{H}{b}\right)\right]
$$

Then:

$$
\begin{aligned}
P & =A\left\{a_{1} \ln (H)+\frac{c_{1}}{2} \ln \left(H-\alpha_{1}\right)^{2}\right. \\
& +\frac{d_{1}}{2} \ln n\left(H-\beta_{1}\right)^{2}+\frac{e_{1}}{2} \ln \left(H^{2}+b^{2}\right\}+\frac{f_{1}}{b} \tan ^{-1}\left(\frac{H}{b}\right) \\
& \left.-\left(\frac{K_{v}}{8 \varepsilon^{6}}\right)\left[a_{1 v} \ln (H)+\frac{e_{1 v}}{2} \ln \left(H^{2}+b^{2}\right)+\frac{f_{1 v}}{b} \tan ^{-1}\left(\frac{H}{b}\right)\right]\right\} \\
& -S \cos (2 \theta)+B
\end{aligned}
$$

Where:

$$
A=\frac{-6 \mu_{i} q \varepsilon}{\pi c^{3} p_{i}}, a_{1}=\frac{1}{\alpha_{1} \beta_{1} b^{2}}, a_{1 v}=\frac{8 \varepsilon^{4}+4 \varepsilon^{2}+3}{b^{2}},
$$




$$
\begin{gathered}
c_{1}=\frac{1}{\alpha_{1}\left(\alpha_{1}-\beta_{1}\right)\left(b^{2}+\alpha_{1}^{2}\right)}, d_{1}=-\frac{1}{\beta_{1}\left(\alpha_{1}-\beta_{1}\right)\left(b^{2}+\alpha_{1}^{2}\right)} \\
e_{1}=\frac{\left(b^{2}-\alpha_{1} \beta_{1}\right)}{b^{2}\left(b^{2}+\alpha_{1}^{2}\right)\left(b^{2}+\beta_{1}^{2}\right)} \\
e_{1 v}=\frac{3\left(6 b^{2}-1\right)+4\left(b^{2}-1\right) \varepsilon^{2}-8 \varepsilon^{4}}{b^{2}} \\
f_{1}=\frac{\alpha_{1}+\beta_{1}}{\left(b^{2}+\alpha_{1}^{2}\right)\left(b^{2}+\beta_{1}^{2}\right)} \quad, f_{1 v}=-4\left(3+2 \varepsilon^{2}\right) \\
\alpha_{1}=(1+\varepsilon), \beta_{1}=(1-\varepsilon)
\end{gathered}
$$

Putting equations (6) into a simple form gives:

$$
P=A Z-S \cos (2 \theta)+B
$$

Applying the boundary conditions:

$$
\begin{gathered}
P=1 \text { at } \theta=\theta_{i} \text { and } P=0 \text { at } \theta=\theta_{e} \\
A=\frac{1+S\left(\cos 2 \theta_{i}-\cos 2 \theta_{e}\right)}{Z_{i}-Z_{e}} \\
B=S \cos 2 \theta_{e}-A Z_{e}
\end{gathered}
$$

Where:

$$
\begin{aligned}
& Z_{i}= a_{1} \ln \left(H_{i}\right)+\frac{c_{1}}{2} \ln \left(H_{i}-\alpha_{1}\right)^{2}+ \\
& \frac{d_{1}}{2} \ln \left(H_{i}-\beta_{1}\right)^{2}+\frac{e_{1}}{2} \ln \left(H_{i}^{2}+b^{2}\right)+\frac{f_{1}}{b} \tan ^{-1}\left(\frac{H_{i}}{b}\right)- \\
&\left(\frac{K_{v}}{8 \varepsilon^{6}}\right)\left[a_{1 v} \ln \left(H_{i}\right)+\frac{e_{1 v}}{2} \ln \left(H_{i}^{2}+b^{2}\right)+\frac{f_{1 v}}{b} \tan ^{-1}\left(\frac{H_{i}}{b}\right)\right] \\
& Z_{e}=a_{1} \ln \left(H_{e}\right)+\frac{c_{1}}{2} \ln \left(H_{e}-\alpha_{1}\right)^{2}+ \\
& \frac{d_{1}}{2} \ln \left(H_{e}-\beta_{1}\right)^{2}+\frac{e_{1}}{2} \ln \left(H_{e}^{2}+b^{2}\right)+\frac{f_{1}}{b} \tan ^{-1}\left(\frac{H_{e}}{b}\right)- \\
&\left(\frac{K_{v}}{8 \varepsilon^{6}}\right)\left[a_{1 v} \ln \left(H_{e}\right)+\frac{e_{1 v}}{2} \ln \left(H_{e}^{2}+b^{2}\right)+\frac{f_{1 v}}{b} \tan ^{-1}\left(\frac{H_{e}}{b}\right)\right]
\end{aligned}
$$

\section{Appendix 1.2. Pressure Distribution ( $\varepsilon=0$ )}

From equations (2-3):

$$
\begin{aligned}
& d P_{1}=\left[\left(\frac{a_{c}}{\left(1+b^{2}\right) \sin \theta}\right)+2 S \sin (2 \theta)\right] d \theta \\
& d P_{v}=\left(\frac{a_{c} K_{v} \sin ^{2} \theta}{\left(1+b^{2}\right) \sin \theta}\right) d \theta
\end{aligned}
$$

The integration of $\left(d P_{1}\right)$ could be easily found in Yacout [4] as:

$$
P_{1}=\frac{A}{1+b^{2}} \ln \left(\frac{\sin \theta}{1+\cos \theta}\right)-S \cos (2 \theta)+B
$$

The integration of $\left(d P_{v}\right)$ is:

$$
P_{v}=\left(\frac{-A K_{v}}{1+b^{2}}\right) \cos \theta
$$

Then:

$$
P=\frac{A}{1+b^{2}}\left[\ln \left(\frac{\sin \theta}{1+\cos \theta}\right)+K_{v} \cos \theta\right]-S \cos (2 \theta)+B
$$

Where:

$$
A=a_{c}
$$

Putting equations into a simple form gives:

$$
P=A Z-S \cos (2 \theta)+B
$$

Applying the boundary conditions:

$$
\begin{gathered}
P=1 \text { at } \theta=\theta_{i} \text { and } P=0 \text { at } \theta=\theta_{e} \\
A=\frac{\left(1+b^{2}\right)\left[1+S\left(\cos 2 \theta_{i}-\cos 2 \theta_{e}\right)\right.}{Z_{i}-Z_{e}} \\
B=S \cos 2 \theta_{e}-\frac{A}{1+b^{2}} Z_{e}
\end{gathered}
$$

Where:

$$
\begin{gathered}
Z_{i}=\ln \left(\frac{\sin \theta_{i}}{\cos \theta_{i}}\right)+K_{v} \cos \theta_{i} \\
Z_{e}=\ln \left(\frac{\sin \theta_{e}}{\cos \theta_{e}}\right)+K_{v} \cos \theta_{e}
\end{gathered}
$$

\section{Appendix 1.3. Pressure Distribution $(\varepsilon=1)$}

From equations (2-3):

$$
\begin{aligned}
& d P_{1}=\left[\left(\frac{a_{c}}{\left(H^{3}+b^{2} H\right) \sin \theta}\right)+2 S \sin (2 \theta)\right] d \theta \\
& d P_{v}=a_{c}\left(\frac{K_{v} \sin ^{2} \theta}{\left(H^{3}+b^{2} H\right) \sin \theta}\right) d \theta
\end{aligned}
$$

The integration of $\left(d P_{1}\right)$ could be easily found in Yacout [4] or in another form as:

$$
\begin{aligned}
P_{1} & =A\left[\frac{1}{2 b^{2} H}-\left(\frac{1}{4 b^{2}}\right) \ln (H)+\frac{1}{4\left(4+b^{2}\right)} \ln (2-H)\right. \\
& \left.+\frac{1}{2 b^{2}\left(4+b^{2}\right)} \ln \left(H^{2}+b^{2}\right)+\frac{2}{b^{3}\left(4+b^{2}\right)} \tan ^{-1}\left(\frac{H}{b}\right)\right] \\
& -S \cos (2 \theta)+B \\
P_{v} & =\left(-A K_{v}\right)\left[a_{v} \ln (H)+\frac{e_{v}}{2} \ln \left(H^{2}+b^{2}\right)\right]
\end{aligned}
$$

Then: 


$$
\begin{aligned}
P & =A\left\{\frac{1}{2 b^{2} H}-\left(\frac{1}{4 b^{2}}\right) \ln (H)+\frac{1}{4\left(4+b^{2}\right)} \ln (2-H)\right. \\
& +\frac{1}{2 b^{2}\left(4+b^{2}\right)} \ln \left(H^{2}+b^{2}\right)+\frac{2}{b^{3}\left(4+b^{2}\right)} \tan ^{-1}\left(\frac{H}{b}\right) \\
& \left.+K_{v}\left[a_{v} \ln (H)+\frac{e_{v}}{2} \ln \left(H^{2}+b^{2}\right)\right]\right\}-S \cos (2 \theta)+B
\end{aligned}
$$

Where:

$$
A=a_{c}, a_{v}=\left(1 / b^{2}\right), e_{v}=-\left(1 / b^{2}\right)
$$

Putting equations into a simple form gives:

$$
P=A Z-S \cos (2 \theta)+B
$$

Applying the boundary conditions:

$$
\begin{gathered}
P=1 \text { at } \theta=\theta_{i} \text { and } P=0 \text { at } \theta=\theta_{e} \\
A=\frac{1+S\left(\cos 2 \theta_{i}-\cos 2 \theta_{e}\right)}{Z_{i}-Z_{e}} \\
B=S \cos 2 \theta_{e}-A Z_{e}
\end{gathered}
$$

Where:

$$
\begin{aligned}
Z_{i} & =\frac{1}{2 b^{2} H_{i}}-\left(\frac{1}{4 b^{2}}\right) \ln \left(H_{i}\right)+\frac{1}{4\left(4+b^{2}\right)} \ln \left(2-H_{i}\right) \\
& +\frac{1}{2 b^{2}\left(4+b^{2}\right)} \ln \left(H_{i}^{2}+b^{2}\right)+\frac{2}{b^{3}\left(4+b^{2}\right)} \tan ^{-1}\left(\frac{H_{i}}{b}\right) \\
& +K_{v}\left[a_{v} \ln \left(H_{i}\right)+\frac{e_{v}}{2} \ln \left(H_{i}{ }^{2}+b^{2}\right)\right] \\
Z_{e} & =\frac{1}{2 b^{2} H_{e}}-\left(\frac{1}{4 b^{2}}\right) \ln \left(H_{e}\right)+\frac{1}{4\left(4+b^{2}\right)} \ln \left(2-H_{e}\right) \\
+ & \frac{1}{2 b^{2}\left(4+b^{2}\right)} \ln \left(H_{e}{ }^{2}+b^{2}\right)+\frac{2}{b^{3}\left(4+b^{2}\right)} \tan ^{-1}\left(\frac{H_{e}}{b}\right) \\
+ & K_{v}\left[a_{v} \ln \left(H_{e}\right)+\frac{e_{v}}{2} \ln \left(H_{e}{ }^{2}+b^{2}\right)\right]
\end{aligned}
$$

\section{Appendix 1.4. Dynamic Pressure Phenomenon}

Equation (6) shows that it includes two terms naturally generate dynamic pressure whether the eccentricity ratio is positive or negative while this phenomenon disappears in the two special cases.

The first term is:

$$
\begin{aligned}
& \frac{c_{1}}{2} \ln \left(H-\alpha_{1}\right)^{2}=c_{1} \ln \left(H-\alpha_{1}\right) \\
& \frac{d_{1}}{2} \ln \left(H-\beta_{1}\right)^{2}=d_{1} \ln \left(H-\beta_{1}\right)
\end{aligned}
$$

Hence:

$$
\begin{array}{ll}
P_{D}=A\left[c_{1} \ln \left(H-\alpha_{1}\right)\right] & \text { for }(+\mathcal{E}) \\
P_{D}=A\left[d_{1} \ln \left(H-\beta_{1}\right)\right] & \text { for }(-\mathcal{E})
\end{array}
$$

\section{Appendix 2.1. Load Carrying Capacity ( $\pm \varepsilon)$}

From Yacout [1-4]:

$$
W=\sin ^{2} \theta_{i}+2 \int_{\theta_{i}}^{\theta_{e}} P \sin \theta \cos \theta d \theta
$$

From Eq. (3) into Eq. (15) gives

$$
W=\sin ^{2} \theta_{i}+2 \int_{\theta_{i}}^{\theta_{e}}\left(P_{1}-P_{v}\right) \sin \theta \cos \theta d \theta W=W_{1}-W_{v}
$$

Where:

$$
\begin{gathered}
W_{1}=\sin ^{2} \theta_{i}+2 \int_{\theta_{i}}^{\theta_{e}} P_{1} \sin \theta \cos \theta d \theta \\
W_{v}=2 \int_{\theta_{i}}^{\theta_{e}} P_{v} \sin \theta \cos \theta d \theta
\end{gathered}
$$

The integration of $\left(W_{1}\right)$ could be easily found in Yacout [4] or in another form as:

$$
\begin{aligned}
W_{1}= & \sin ^{2} \theta_{i}+2 A\left[a_{1} w_{1}+c_{1} w_{2}+d_{1} w_{3}+\frac{e_{1}}{2} w_{4}+\frac{f_{1}}{b} w_{5}\right] \\
& +\frac{S}{4}\left(\cos ^{2} 2 \theta_{e}-\cos ^{2} 2 \theta_{i}\right)+B\left(\sin ^{2} \theta_{e}-\sin ^{2} \theta_{i}\right)
\end{aligned}
$$

Where:

$$
\begin{aligned}
w_{1}= & \frac{1}{2 \varepsilon^{2}}\left[H(2-H) \ln (H)+\frac{H(H-4)}{2}\right]_{\theta_{i}}^{\theta_{o}} \\
w_{2}= & \left(\frac{-1}{4 \varepsilon^{2}}\right)\left[\left(H-\alpha_{1}\right)\left(H-\beta_{1}\right) \ln \left(H-\alpha_{1}\right)^{2}\right. \\
& \left.-\left(H-\alpha_{1}\right)\left(H+3 \alpha_{1}-4\right)\right]_{\theta_{i}}^{\theta_{o}} \\
w_{3}= & \left(\frac{-1}{4 \varepsilon^{2}}\right)\left[\left(H-\alpha_{1}\right)\left(H-\beta_{1}\right) \ln \left(H-\beta_{1}\right)^{2}\right. \\
& \left.-\left(H-\beta_{1}\right)\left(H+3 \beta_{1}-4\right)\right]_{\theta_{i}}^{\theta_{o}} \\
w_{4}= & \left(\frac{1}{4 \varepsilon^{2}}\right)\left[\left(2 H-H^{2}-b^{2}\right) \ln \left(H^{2}+b^{2}\right)\right. \\
& +4 b \tan { }^{-1}\left(\frac{H}{b}\right)+H^{2}-4 H_{\theta_{i}}^{\theta_{o}} \\
w_{5}= & \left(\frac{-1}{2 b \varepsilon^{2}}\right)\left\{\left(H^{2}-2 H+b^{2}\right) \tan ^{-1}\left(\frac{H}{b}\right)\right. \\
& \left.+b\left[\ln \left(H^{2}+b^{2}\right)-(H)\right]\right\}_{\theta_{i}}^{\theta_{o}}
\end{aligned}
$$

The integration of $\left(W_{v}\right)$ is:

$$
W_{v}=\left(\frac{-A K_{v}}{\varepsilon^{2}}\right)\left[a_{1 v} I_{1}+\frac{e_{1 v}}{2} I_{2}\right]
$$

Where:

$$
I_{1}=\left(\frac{H}{2}\right)\left[(H-2) \ln (H)-\frac{H-4}{2}\right]
$$




$$
\begin{aligned}
I_{2}= & \left(\frac{H^{2}-2 H+b^{2}}{2}\right) \ln \left(H^{2}+b^{2}\right)-2 b \tan ^{-1}\left(\frac{H}{b}\right) \\
& \left.-\frac{H-4 H}{2}\right] \\
W & =\sin ^{2} \theta_{i}+2 A\left[\left(a_{1} w_{1}+c_{1} w_{2}+d_{1} w_{3}+\frac{e_{1}}{2} w_{4}+\frac{f_{1}}{b} w_{5}\right)\right. \\
& \left.-\left(\frac{-K_{v}}{\varepsilon^{2}}\right)\left(a_{1 v} I_{1}+\frac{e_{1 v}}{2} I_{2}\right)\right]_{\theta_{i}}^{\theta_{o}}+\frac{S}{4}\left[\cos ^{2} 2 \theta\right]_{\theta_{i}}^{\theta_{o}}+B\left[\sin ^{2} \theta\right]_{\theta_{i}}^{\theta_{o}}
\end{aligned}
$$

\section{Appendix 2.2. Load Carrying Capacity $(\varepsilon=0)$}

From equations $(8,9)$ into $(16)$ :

$$
\begin{aligned}
W_{1}= & \sin ^{2} \theta_{i} \\
+ & 2 \int_{\theta_{i}}^{\theta_{e}}\left[\frac{A}{1+b^{2}} \ln \left(\frac{\sin \theta}{1+\cos \theta}\right)-S \cos (2 \theta)+B\right] \\
& x(\sin \theta \cos \theta) d \theta \\
W_{v}= & 2 \int_{\theta_{i}}^{\theta_{e}}\left[\left(\frac{-A K_{v}}{1+b^{2}}\right) \cos \theta\right] \sin \theta \cos \theta d \theta
\end{aligned}
$$

The integration of $\left(W_{1}\right)$ could be found in Yacout [4] as:

$$
\begin{aligned}
W_{1}= & \sin ^{2} \theta_{l}+\left(\frac{A}{1+b^{2}}\right)\left[\sin ^{2} \theta \ln \left(\frac{\sin \theta}{1+\cos \theta}\right)+\cos \theta-\frac{1}{2}\right]_{\theta_{i}}^{\theta_{o}} \\
& +\frac{S}{4}[\cos (2 \theta)]_{\theta_{i}}^{\theta_{o}}+B\left[\sin ^{2} \theta\right]_{\theta_{i}}^{\theta_{o}}
\end{aligned}
$$

The integration of $\left(W_{v}\right)$ is:

$$
W_{v}=\frac{2 A K_{v}}{3\left(1+b^{2}\right)}\left[\cos ^{3}(2 \theta)\right]_{\theta_{i}}^{\theta_{o}}
$$

Then:

$$
\begin{aligned}
& W=\sin ^{2} \theta_{l}+\left(\frac{A}{1+b^{2}}\right)\left[\sin ^{2} \theta \ln \left(\frac{\sin \theta}{1+\cos \theta}\right)+\cos \theta-\frac{1}{2}\right. \\
& \left.-\left(\frac{2}{3}\right) K_{v} \cos ^{3}(2 \theta)\right]_{\theta_{i}}^{\theta_{o}}+\frac{S}{4}[\cos (2 \theta)]_{\theta_{i}}^{\theta_{o}}+B\left[\sin ^{2} \theta\right]_{\theta_{i}}^{\theta_{o}}
\end{aligned}
$$

\section{Appendix 2.3. Load Carrying Capacity $(\varepsilon=1)$}

From equations $(11,12)$ into $(16)$ :

$$
\begin{aligned}
& W_{1}=\sin ^{2} \theta_{i} \\
& +2 \int_{\theta_{i}}^{\theta_{e}}\left\{A \left[\frac{1}{2 b^{2} H}-\left(\frac{1}{4 b^{2}}\right) \ln (H)+\frac{1}{4\left(4+b^{2}\right)} \ln (2-H)\right.\right. \\
& \left.+\frac{1}{2 b^{2}\left(4+b^{2}\right)} \ln \left(H^{2}+b^{2}\right)+\frac{2}{b^{3}\left(4+b^{2}\right)} \tan ^{-1}\left(\frac{H}{b}\right)\right] \\
& -S \cos (2 \theta)+B\}(\sin \theta \cos \theta) d \theta \\
& W_{v}=-2 A K_{v} \int_{\theta_{i}}^{\theta_{r}}\left[a_{v} \ln (H)+\frac{e_{v}}{2} \ln \left(H^{2}+b^{2}\right)\right] \\
& x(\sin \theta \cos \theta) d \theta
\end{aligned}
$$

The integration of $\left(W_{1}\right)$ could be easily found in Yacout [4] or in another form as:

$$
\begin{aligned}
W_{1}= & \sin ^{2} \theta_{i}+2 A\left[a_{1} w_{1}+c_{1} w_{2}+d_{1} w_{3}+e_{1} w_{4}+f_{1} w_{5}\right]_{\theta_{i}}^{\theta_{o}} \\
& +\frac{S}{4}[\cos (2 \theta)]_{\theta_{i}}^{\theta_{o}}+B\left[\sin ^{2} \theta\right]_{\theta_{i}}^{\theta_{o}}
\end{aligned}
$$

The integration of $\left(W_{v}\right)$ is:

$$
W_{v}=-2 A K_{v}\left[a_{v} I_{1 v}+\frac{e_{v}}{2} I_{2 v}\right]
$$

Then:

$$
\begin{aligned}
W= & \sin ^{2} \theta_{i}+2 A\left\{a_{1} w_{1}+a_{2} w_{2}+a_{3} w_{3}+a_{4} w_{4}+a_{5} w_{5}\right. \\
& \left.-K_{v}\left[a_{v} I_{1 v}+\frac{e_{v}}{2} I_{2 v}\right]\right\}_{\theta_{i}}^{\theta_{o}}+\frac{S}{4}[\cos (2 \theta)]_{\theta_{i}}^{\theta_{o}}+B\left[\sin ^{2} \theta\right]_{\theta_{i}}^{\theta_{o}}
\end{aligned}
$$

Where:

$$
\begin{gathered}
a_{1}=\left(1 / 2 b^{2}\right), a_{2}=-\left(1 / 4 b^{2}\right), a_{3}=1 / b^{2}\left(4+b^{2}\right) \\
a_{4}=1 / 2 b^{2}\left(4+b^{2}\right), a_{5}=1 / b^{3}\left(4+b^{2}\right), a_{v}=\left(1 / b^{2}\right) \\
e_{v}=-\left(1 / b^{2}\right) \\
w_{1}=\ln (H)-H+1 \\
w_{3}=\left[\frac{2 H-H^{2}}{2} \ln (2-H)\right]-\left[\frac{(2-H)(2+H)}{2}\right] \\
w_{4}=\left[\frac{2 H-H^{2}-b^{2}}{2} \ln \left(H^{2}+b^{2}\right)\right]+2 b \tan ^{-1}\left(\frac{H}{b}\right)+\left[\frac{H(H-4)+b^{2}}{2}\right] \\
w_{5}=\left[\frac{2 H-H^{2}-b^{2}}{2} \tan ^{-1}\left(\frac{H}{b}\right)\right]+\frac{b}{2}\left[H-\ln \left(H^{2}+b^{2}\right)\right]
\end{gathered}
$$

Equations ( $6 \& 19$ ) could be easily applied to the positive and negative eccentricity ratios (with exceptions of both the zero and the unity eccentricity ratios) avoiding the mathematical difficulties and complications in Yacout [4].

\section{Appendix 2.4. Dynamic Load Phenomenon}

The integration of the dynamic pressure generates the dynamic load.

From equation (12):

$$
\begin{array}{ll}
L_{d}=2 A\left(c_{1} w_{2}\right) & \text { for }(+\varepsilon) \\
L_{d}=2 A\left(d_{1} w_{3}\right) & \text { for }(-\varepsilon)
\end{array}
$$

\section{Appendix 3. Temperature Distribution}

From Yacout [2]:

$$
\begin{aligned}
& \frac{d T}{d \theta}=\left(\frac{p_{1}}{\rho c_{v}}\right)\left[\left(\frac{d P}{d \theta}\right)^{2}-2 S \sin (2 \theta)\left(\frac{d P}{d \theta}\right)+\frac{(S \sin 2 \theta)^{2}}{0.84}\right. \\
& \left.+12\left(\frac{\mu \Omega R^{2} \sin \theta}{p_{i} h^{2}}\right)^{2}\right]\left(S \sin 2 \theta-\frac{d P}{d \theta}\right)^{-1}=X
\end{aligned}
$$


For applying the energy equation to the recessed clearance type of bearings the term $12\left(\frac{\mu \Omega R^{2} \sin \theta}{p_{i} h^{2}}\right)^{2}$ of the equation could be put on the following form:

$$
G\left[\frac{\sin ^{2} \theta\left(1-K_{v} \sin ^{2} \theta\right)}{(1+\varepsilon \cos \theta)^{4}}\right]
$$

Where:

$$
\begin{aligned}
& G=\left(\frac{160 S \mu_{i}^{2}}{\rho \beta p_{s} R^{2} K_{c}^{4}}\right) \\
& \mu=\mu_{i}\left(1-K_{v} \sin ^{2} \theta\right) \\
& p_{i}=\beta p_{s} \\
& S=\frac{3}{40} \frac{\rho \Omega^{2} R^{2}}{p_{i}}
\end{aligned}
$$

Then the temperature rise and distribution could be numerically determined as:

$$
\begin{aligned}
& \frac{\Delta T}{\Delta \theta}=X \\
& T_{n}=T_{n-1}+X \Delta \theta \\
& T_{1}=T_{i}
\end{aligned}
$$

\section{Appendix 4. Ffrictional Factor Equation}

\section{Appendix 4.1. The Case of $( \pm \varepsilon)$}

Yacout [1-4]:

$$
m_{o}=2 \pi \mu \Omega R^{4} \int_{\theta_{i}}^{\theta_{o}} \frac{h_{o}^{2}+\sigma_{o}^{2}}{h_{o}^{3}} \sin ^{3} \theta d \theta
$$

For variable viscosity:

$$
m_{o}=2 \pi \mu_{i} \Omega R^{4} \int_{\theta_{i}}^{\theta_{o}} \frac{h_{o}^{2}+\sigma_{o}^{2}}{h_{o}^{3}} \sin ^{3} \theta\left(1-K_{v} \sin ^{2}\right) d \theta
$$

Introducing the dimensionless groups:

$$
\begin{gathered}
H=\frac{h_{o}}{c} \sigma=\frac{\sigma_{o}}{c}, M=\frac{m_{o} c}{2 \pi \mu_{i} \Omega R^{4}} \\
M=\int_{\theta_{i}}^{\theta_{o}} \frac{H^{2}+\sigma^{2}}{H^{3}} \sin ^{3} \theta\left(1-K_{v} \sin ^{2}\right) d \theta \\
M=\int_{\theta_{i}}^{\theta_{o}} \frac{H^{2}+\sigma^{2}}{H^{3}} \sin ^{3} \theta d \theta-K_{v} \int_{\theta_{i}}^{\theta_{\theta}} \frac{H^{2}+\sigma^{2}}{H^{3}} \sin ^{5} \theta d \theta \\
M_{1}=\int_{\theta_{i}}^{\theta_{o}} \frac{H^{2}+\sigma^{2}}{H^{3}} \sin ^{3} \theta d \theta \\
M_{v}=K_{v} \int_{\theta_{i}}^{\theta_{0}} \frac{H^{2}+\sigma^{2}}{H^{3}} \sin ^{5} \theta d \theta \\
M=M_{1}-M_{v}
\end{gathered}
$$

The integration of $\left(M_{1}\right)$ could be easily found in Yacout [4] or in another form as:

$$
\begin{aligned}
M_{1} & =\frac{1}{\varepsilon^{3}}\left[\frac{(H)^{2}}{2}-2(H)+\left(\alpha_{1} \beta_{1}+\sigma^{2}\right) \ln (H)+\frac{2 \sigma^{2}}{H}\right. \\
& \left.-\frac{\alpha_{1} \beta_{1} \sigma^{2}}{2 H^{2}}\right]_{\theta_{i}}^{\theta_{o}}
\end{aligned}
$$

The integration of $\left(M_{v}\right)$ is:

$$
\begin{aligned}
M_{v} & =\left(\frac{-K_{v}}{\varepsilon^{5}}\right)\left[\frac{H^{4}}{4}-\frac{4 H^{3}}{3}+\frac{\left(6+\sigma^{2}-2 \varepsilon^{2}\right) H^{2}}{2}\right. \\
& -4\left(\alpha_{1} \beta_{1}+\sigma^{2}\right) H+\left(\alpha_{1}^{2} \beta_{1}^{2}+6 \sigma^{2}-2 \varepsilon^{2} \sigma^{2}\right) \ln (H) \\
& \left.-\frac{4 \alpha_{1} \beta_{1} \sigma^{2}}{H}-\frac{\alpha_{1}^{2} \beta_{1}^{2} \sigma^{2}}{2 H^{2}}\right]_{\theta_{i}}^{\theta_{o}}
\end{aligned}
$$

Appendix 4.2. The First exceptional case $(\varepsilon=0)$

As $(\varepsilon=0)$ equation (24) becomes:

$$
\begin{aligned}
& M_{1}=\left(1+\sigma^{2}\right) \int_{\theta_{i}}^{\theta_{0}} \sin ^{3} \theta d \theta \\
& M_{v}=K_{v}\left(1+\sigma^{2}\right) \int_{\theta_{i}}^{\theta_{e}} \sin ^{5} \theta d \theta
\end{aligned}
$$

The integration of $\left(M_{1}\right)$ could be found in Yacout [4] as:

$$
M_{1}=\left(1+\sigma^{2}\right)\left[\frac{\cos ^{3} \theta}{3}-\cos \theta\right]_{\theta_{i}}^{\theta_{o}}
$$

The integration of $\left(M_{v}\right)$ is:

$$
M_{v}=\left(-K_{v}\right)\left(1+\sigma^{2}\right)\left[\cos \theta-\frac{2 \cos ^{3} \theta}{3}+\frac{\cos ^{5} \theta}{5}\right]_{\theta_{i}}^{\theta_{o}}
$$

Then:

$$
M=M_{1}-M_{v}
$$

Appendix 4.3. The Second exceptional case $(\varepsilon=1)$

As $(\varepsilon=1)$ equation (25) could be directly applied to this exceptional case where its form changes to be:

$$
M_{1}=\left[\frac{H^{2}}{2}-2 H+\sigma^{2} \ln (H)+\frac{2 \sigma^{2}}{H}\right]_{\theta_{i}}^{\theta_{i}}
$$

As in Yacout [4]

$$
\begin{gathered}
M_{v}=\left(-K_{v}\right)\left[\frac{H^{4}}{4}-\frac{4 H^{3}}{3}+\frac{\left(4+\sigma^{2}\right) H^{2}}{2}\right. \\
\left.-4 \sigma^{2} H+4 \sigma^{2} \ln (H)\right]_{\theta_{i}}^{\theta_{o}} \\
M=M_{1}-M_{v}
\end{gathered}
$$

\section{Appendix 5. Stiffness Factor}

From Yacout [4]:

$\beta=\frac{2 K_{c a p}}{\pi c^{3} Q}$ for capillary restrictor 
$\beta=\frac{6 \mu K_{o}}{\pi c^{3} Q \sqrt{3 p_{s}}}$ for orifice restrictor

And through an infinitesimal change of the eccentricity and applying the finite difference method:

$(\bar{\beta})$ And $(\bar{W})$ are numerically calculated; hence:

$$
\begin{aligned}
& S F_{c}=-(\bar{\beta} W+\beta \bar{W}) \\
& S F_{o}=-(\bar{\beta} W+\beta \bar{W})
\end{aligned}
$$

\section{Nomenclature}

$A= \pm\left(6 \mu q \varepsilon / c^{3} \pi p_{i}\right)$

$a=$ Bearing projected area $\left(\pi R^{2}\right)$

abs ( ) = Absolute value of.

$C=-(6 \mu q / \pi)$.

$C_{d}=$ Restrictor Discharge coefficient.

$c=$ Radial clearance.

$c_{v}=$ Lubricant specific heat

$d_{c}=$ Capillary tube diameter.

$d_{o}=$ Orifice restrictor diameter.

$d_{i o}=$ Bearing inlet orifice diameter.

$E(f)=$ Expected value of.

$e=$ Eccentricity.

$F=$ Dimensionless friction factor.

$f=$ Friction factor.

$H=$ Dimensionless film thickness.

$h=$ Film thickness.

$h_{f}=$ Power factor.

$h_{o}=$ Deterministic (mean) part of the film thickness=

$c(1+\varepsilon \cos \theta)$.

$h_{s t}=$ Random stochastic part of the film thickness.

$K_{\mathrm{c}}=c / R$

$K_{\mathrm{e}}=e / R$

$K_{\mathrm{v}}=$ Variable viscosity constant

$l_{c}=$ Capillary tube length.

$L_{D}=$ Dimensionless dynamic load

$M=$ Dimensionless frictional torque $\left(\frac{m_{o} c}{2 \pi \mu \Omega R^{4}}\right)$.

$m=$ Frictional torque.

$m_{o}=$ Deterministic part of the frictional torque.

$m_{s t}=$ Random stochastic part of the frictional torque.

$N=$ Shaft speed (rps).

$P=$ Dimensionless pressure $\left(p / p_{i}\right)$.

$p=$ Mean pressure along the film thickness.

$p_{i}=$ Inlet pressure.
$P_{D}=$ Dynamic pressure

$p_{s}=$ Supply pressure

$p_{d}=$ Power dissipated by the bearing $\left(q \cdot p_{i}\right)$.

$p_{p}=$ Pump power $\left(q \cdot p_{s}\right)$.

$p_{f}=$ Frictional power $(\Omega . m)$.

$p_{t}=$ Total losses.

$Q=$ Dimensionless volume flow rate $= \pm$ A.

$q=$ Volume flow rate through the restrictor or the bearing

$R=$ Bearing radius.

$R e_{c}=$ Reynolds number for the capillary tube flow.

$R e_{o}=$ Reynolds number for the orifice restrictor flow.

$\mathrm{S}=$ Speed parameter $=\left(\frac{3}{40} \frac{\rho \Omega^{2} R^{2}}{p_{i}}\right)$.

$S F_{c}=$ Stiffness factor for capillary tube restrictor.

$S F_{0}=$ Stiffness factor for orifice restrictor.

$\mathrm{T}=$ Temperature

$T_{R}=$ Temperature rise

$W=$ Dimensionless load carrying capacity $\left(w / \pi R^{2} p_{i}\right)$

$\mathcal{W}=$ Load carrying capacity.

$\beta=$ Central pressure ratio $\left(p_{i} / p_{s}\right)$.

$\mathcal{E}=$ Eccentricity ratio $(e / c)$

$\eta=2 \phi_{b} / \pi$

$\boldsymbol{\theta}=$ Angle co-ordinate.

$\theta_{i}=$ Inlet flow angle.

$\theta_{\mathrm{o}}=$ Outlet flow angle.

$\Lambda=\left(h_{\min } / \sigma\right)=$ Dimensionless film thickness parameter

(5 to 100) for hydrodynamic lubrication regime

$\rho=$ Lubricant density

$\sigma=$ Roughness parameter.

$\sigma_{o}^{2}=$ Variance of the film thickness.

$\phi_{b}=$ Seat outer rim angle.

$\phi_{i}=$ Inlet orifice angle.

$\lambda=$ Bearing stiffness.

$\mu=\mu_{o}\left(1-\sin ^{2} \theta\right)=$ Lubricant viscosity

$\xi=$ Normalized roughness parameter.

$\Omega=$ Rotational speed.

Suffixes

Fit $=$ fitted type of bearings.

$c l=$ clearance type of bearings.

\section{References}

[1] Ahmad W. Yacout, Ashraf S. Ismaeel, Sadek Z. Kassab, 2007, "The combined effects of the centripetal inertia and the surface roughness on the hydrostatic thrust spherical bearing performance", Tribolgy International Journal, Vol. 40, No. 3, $522-532$. 
[2] Ahmad W. Yacout Elescandarany" The Effect of the Fluid Film Variable Viscosity on the Hydrostatic Thrust Spherical Bearing Performance in the Presence of Centripetal Inertia and Surface Roughness (Part 1, un-recessed fitted bearing); International journal of Mechanical Engineering and Applications 2018, Vol. 6, No. 1, pp. 1-12.

[3] Ahmad Waguih Yacout Elescandarany" The Effect of the Fluid Film Variable Viscosity on the Hydrostatic Thrust Spherical Bearing Performance in the Presence of Centripetal Inertia and Surface Roughness (Part 2, recessed fitted bearing). International journal of Mechanical Engineering and Applications 2018, Vol. 6, No. 3, pp. 73-90.

[4] Ahmad W. Yacout, Ismail, A. S. and Kassab, S. Z.," The Surface Roughness Effect on the Hydrostatic Thrust Spherical Bearings Performance (Part 2 un-recessed clearance type of bearings), 2006, "ASME International Mechanical Engineering Congress and Exposition, Paper No. IMECE 2006-13004.

[5] Woonsil Lee, Chi Hyuk, An Sung Lee, "A High Bearing Temperature Troubleshooting of Centrifugal Heat-Pump Compressor", $45^{\text {th }}$ Turbo-machinery $\& 32^{\text {nd }}$ pump symposia, Huston, Texas, 12-15, 2016, George R. Brown Convention Center.

[6] Yongping SUN, Minghui HAO, Baoyu SONG, "Bearing capacity and temperature rise characteristics analysis of herringbone groove hydrodynamic lubricating bearing", $2^{\text {nd }}$ International Forum on Electrical Engineering and Automation (IFEEA 2015).

[7] Srinivasan V. "Analysis of Static and Dynamic Load on the Hydrostatic Bearing with Variable Viscosity Affected by the Environmental Temperature", Journal of Environmental Research and Development 2012, Vol. 7, No. 1A, 346-353.

[8] Srinivasan V. "Analysis of Static and Dynamic Load on Hydrostatic Bearing with Variable Viscosity and Pressure", Indian Journal of Science and Technology 2013, Vol. 6 (6s), 4777-4782.

[9] Shigang Wang, Xianfeng Du, Mingzhu Li, Zhongliang Cao,
Jianjia Wang, Analysis of temperature effect on the lubricating state of hydrostatic bearing, Journal of Theoretical and Applied Information Technology 2013, Vol. 48, No. 2, 817821.

[10] Xibing Li, Xun Wang, Ming Li, Yunshi Ma and Ying Huang, The Research Status and Progress of Heavy/Large Hydrostatic Thrust Bearing, Hidawi Publishing Corporation, Advances in Mechanical Engineering, volume 2014, Article ID: 982584. http://dx.doi.org/10.1155/2014/982584.

[11] N. B. Naduvinamani and Archana K. Kadadi, 2013, "The effect of viscosity variation on the micro-polar fluid squeeze film lubrication of a short journal bearing", Advances of Tribology Journal, Vol. 2013, Article ID 743987.

[12] B. Bouchehit, B. Bou-Saïd and M Garcia, 2016, "Static and dynamic performances of refrigerant-lubricated foil bearings", 7th international conference on advanced concepts in mechanical engineering.

[13] Yuan Kang, Jian-Lin Lee, Hua-Chin Huang and Ching-Chu Huang," Design for static stiffness of hydrostatic plain bearings: Constant compensations", Journal of Industrial Lubrication and Tribology 2011, Vol. 63, No. 3, pp: 178-191.

[14] G Jaya Chandra Reddy, C Eswara Reddy, K Rama Krishna Prasad," Effect of viscosity variation on the squeeze film performance of a narrow hydrodynamic journal bearing operating with couple stress fluids", Proceedings of the Institution of Mechanical Engineers, Part J: Journal of Engineering Tribology 2008, Vol. 222, No. 2, pp: 141-150.

[15] Jun Sun, Xinlong Zhu, Liang Zhang, Xianyi Wang, Chunmei Wang, Hu Wang and Xiaoyong Zhao," Effect of surface roughness, viscosity-pressure relationship and elastic deformation on lubrication performance of misaligned journal bearings", Journal of Industrial Lubrication and Tribology 2014 Vol. 66, · No. 3, pp: 337-345.

[16] Chuan-Chieh, Sheng-Yen Hu, Chao-Ping Huang and Yuan Kang, "Influences of Lubricant Viscosity Ratio on Static Characteristics of Hydrostatic Thrust Bearing", MATEC Web of Conferences 2017, 114, 01006. 